\title{
Building a model: developing genomic resources for common milkweed (Asclepias syriaca) with low coverage genome sequencing
}

Shannon CK Straub ${ }^{1 *}$, Mark Fishbein², Tatyana Livshultz ${ }^{3}$, Zachary Foster ${ }^{1}$, Matthew Parks ${ }^{1}$, Kevin Weitemier ${ }^{1}$, Richard C Cronn ${ }^{4}$ and Aaron Liston ${ }^{1}$

\begin{abstract}
Background: Milkweeds (Asclepias L.) have been extensively investigated in diverse areas of evolutionary biology and ecology; however, there are few genetic resources available to facilitate and compliment these studies. This study explored how low coverage genome sequencing of the common milkweed (Asclepias syriaca L.) could be useful in characterizing the genome of a plant without prior genomic information and for development of genomic resources as a step toward further developing A. syriaca as a model in ecology and evolution.

Results: A $0.5 \times$ genome of A. syriaca was produced using Illumina sequencing. A virtually complete chloroplast genome of 158,598 bp was assembled, revealing few repeats and loss of three genes: accD, clpP, and ycf1. A nearly complete rDNA cistron (18S-5.8S-26S; 7,541 bp) and 5S rDNA (120 bp) sequence were obtained. Assessment of polymorphism revealed that the rDNA cistron and $5 \mathrm{~S}$ rDNA had $0.3 \%$ and $26.7 \%$ polymorphic sites, respectively. A partial mitochondrial genome sequence $(130,764 \mathrm{bp})$, with identical gene content to tobacco, was also assembled. An initial characterization of repeat content indicated that Ty1/copia-like retroelements are the most common repeat type in the milkweed genome. At least one A. syriaca microread hit $88 \%$ of Catharanthus roseus (Apocynaceae) unigenes (median coverage of $0.29 x$ ) and $66 \%$ of single copy orthologs (COSII) in asterids (median coverage of $0.14 \times$ ). From this partial characterization of the A. syriaca genome, markers for population genetics (microsatellites) and phylogenetics (low-copy nuclear genes) studies were developed.

Conclusions: The results highlight the promise of next generation sequencing for development of genomic resources for any organism. Low coverage genome sequencing allows characterization of the high copy fraction of the genome and exploration of the low copy fraction of the genome, which facilitate the development of molecular tools for further study of a target species and its relatives. This study represents a first step in the development of a community resource for further study of plant-insect co-evolution, anti-herbivore defense, floral developmental genetics, reproductive biology, chemical evolution, population genetics, and comparative genomics using milkweeds, and A. syriaca in particular, as ecological and evolutionary models.
\end{abstract}

\section{Background}

The advent of next generation sequencing technology has presented the opportunity to obtain genome-scale data for any organism [1-3]. Detailed information about the characteristics of the genome of a non-model organism, especially the high-copy fraction, can be obtained even with very low overall coverage of a genome [4-6].

\footnotetext{
* Correspondence: straubs@science.oregonstate.edu

'Department of Botany and Plant Pathology, Oregon State University, 2082

Cordley Hall, Corvallis, Oregon 97331, USA Full list of author information is available at the end of the article
}

Repeat types can be characterized to assess the presence and prevalence of retrotransposons, DNA transposons, and other simple and low-complexity repeats [e.g., $[4,7,8]]$. Nearly complete sequences of organellar genomes are also readily obtained [e.g., [4,9-14]]. If genomic resources are available for a close relative, a portion of the gene space of the nuclear genome might also be surveyed [12]. In contrast to the above studies, next generation sequencing in non-model organisms has thus far primarily been used for characterization of transcriptomes [3] and as a tool for marker development in
C Biomed Central 
population and conservation genetics [e.g., [15,16]], phylogenetics $[10,17]$, and other areas of evolutionary biology [e.g., [18]].

As the most intensively studied member of a genus that has been investigated extensively in diverse areas of evolutionary biology and ecology [e.g., [19-24]], the common milkweed (Asclepias syriaca L., Apocynaceae) is an attractive non-model target for the development of genomic resources. Interest in the genus has been focused on complex and specialized floral structures, particularly as they relate to insect pollination [21], and on the remarkable chemical ecology exemplified by cardenolide-sequestering specialist herbivores, such as the monarch butterfly (Danaus plexippus), that utilize Asclepias defense compounds to defend themselves against their own predators [25]. In the context of floral biology and plant-insect coevolution, Asclepias has the potential to add substantially to existing ecological and evolutionary model systems (e.g., Arabidopsis, Mimulus, Aquilegia), although genomic resources for Asclepias are currently far more limiting than for these model systems. Genomic resources have been developed for only two other species in the same order (Gentianales) as Asclepias: coffee (Coffea; http://www.coffeegenome.org) and Madagascar periwinkle (Catharanthus roseus: Apocynaceae), for which there are expressed sequence tag (EST) collections [26-28].

The aims of this study were to demonstrate how a small amount of Illumina short read sequence data could be useful for whole genome characterization of a plant species with no prior genomic information and to develop genomic resources for milkweeds. To these ends, the genome of Asclepias syriaca was characterized using $0.5 \times$ sequence coverage obtained from a single lane of 40 bp Illumina reads. Reference-guided assembly was used to assemble and characterize the high copy fraction of the A. syriaca genome, including the complete chloroplast and partial mitochondrial genomes and a nearly complete nuclear ribosomal DNA cistron and $5 \mathrm{~S}$ rDNA sequence. De novo assemblies were used to characterize the repeat structure of the genome and to develop nuclear markers consisting of low-copy nuclear loci for phylogenetics and microsatellite loci for population genetics. This study highlights the extent to which a non-model plant genome can be characterized with low coverage genome sequencing and represents the first step in developing the resources needed to develop Asclepias as a new genetic model system in ecological and evolutionary studies [29].

\section{Methods}

\section{Genome size estimation}

Leaves were collected from five A. syriaca individuals from an experimental agricultural population in western
Illinois and frozen at $-20^{\circ} \mathrm{C}$. Samples of approximately $3 \times 3 \mathrm{~mm}$ green leaf tissue were chopped in $150 \mu \mathrm{l}$ Partec CysStain UV Precise P Nuclei extraction buffer (made $1.0 \%$ w/v PVP-40; Partec North America, Inc., Swedesboro, NJ, USA) on ice in the presence of $(\sim 8 \times 8 \mathrm{~mm}) \mathrm{B}$ 73 maize $(1 \mathrm{C}=2.725 \mathrm{pg})$ leaf tissue as internal reference. Upon filtering and addition of $600 \mu \mathrm{l} \mathrm{CysStain} \mathrm{UV}$ Precise P Staining Buffer, samples were run on a Partec PA flow cytometer until at least 1000 counts were recorded for both the sample and reference peaks. The estimated DNA content values for the five individuals were averaged and the genome size calculated using a standard conversion factor [30].

\section{Illumina library preparation and sequencing}

Leaf tissue was sampled from a single individual from a natural population of $A$. syriaca in Ogle County, Illinois [Fishbein 4885 (OKLA)]. Total genomic DNA was extracted using the Wizard ${ }^{\circledR}$ Genomic DNA Purification kit (Promega, Madison, WI, USA) following homogenization of $100 \mathrm{mg}$ of silica-dried tissue flash-frozen in liquid $\mathrm{N}_{2}$ and ground with mortar and pestle. A BioRuptor Sonicator (Diagenode Inc., Denville, NJ, USA) was used to shear $2.5 \mu \mathrm{g}$ of the extracted DNA using 15 one min cycles (30 sec on $/ 30 \mathrm{sec}$ off, setting 'high', $4^{\circ} \mathrm{C}$ ). Subsequent library preparation followed the standard multi-step Illumina protocol [31]. Ligated, size-selected fragments ( 280 - $320 \mathrm{bp})$ were amplified through 18 cycles of polymerase chain reaction (PCR), using Phusion High-Fidelity PCR Master Mix (New England Biolabs, Ipswich, MA, USA) and standard Illumina primers. Resulting product concentrations were determined using a Nanodrop 1000 (ThermoFisher Scientific, Wilmington, DE, USA).

Enriched, cleaned product was diluted to $5 \mathrm{pM}$ and submitted for sequencing on an Illumina GAII sequencer at the Center for Genome Research and Biocomputing (CGRB) at Oregon State University (Corvallis, OR, USA). Denaturation, cluster generation, and subsequent sequencing followed the manufacturer's recommendations. Sequencing was completed over 40 cycles on a single lane of the Illumina GAII. Image analysis, basecalling and error estimation were performed using the Illumina GA Pipeline version 1.5. Images were collected from 120 tiles, averaging over 107,000 clusters/tile, and resulted in over $420 \mathrm{Mbp}$ of sequence data after quality filtering.

\section{Reference-guided assembly}

Reference-guided assembly of microreads was facilitated using a pipeline of five open source scripts called "alignreads." The pipeline incorporates: YASRA [32], a short read assembler; NUCMER, an aligner from the MUMmer 3.0 suite [33,34]; delta-filter, a utility program from 
the MUMmer 3.0 suite that removes excessive/erroneous alignments; "sumqual," a python script created to integrate the NUCMER alignment information with the YASRA assembly information; and "qualtofa," another python script that reformats and filters the output of sumqual based on user inputs. A consensus sequence of the aligned contigs is produced, which can be masked based on base call proportion and coverage depth; single nucleotide polymorphisms (SNPs) can be masked similarly using separate user specifications. Scripts are available for download at http://milkweedgenome.org. Read depth was determined by calculating median base sequencing depth from the alignreads output.

\section{Chloroplast Genome}

The reference-guided assembly of the chloroplast genome was completed in alignreads using the chloroplast genome sequence of oleander (Nerium oleander: Apocynaceae; M. Moore and D. Soltis, unpublished data; see [17] for coding sequences) with only one copy of the inverted repeat included as the reference and no masking. A second alignment of the YASRA contigs was completed using Mulan [35], and the resulting consensus sequence was compared to the alignreads output and tested as an alternate reference input for alignreads. All of the consensus sequences were aligned to each other and the oleander reference using MAFFT 6.240 [36]. A preliminary annotation of the A. syriaca chloroplast genome was completed using DOGMA [37]. The annotations of protein coding regions, ribosomal DNA (rDNA), and sequences corresponding to transfer RNAs (tRNAs) were improved by fixing annotation and assembly mistakes (e.g., no start or stop codon, internal stop codons, frameshifts, endpoints of tRNA sequences) through examination of the actual assembly of the short reads at that location in Tablet vers. 1.10.09.20 [38] and/or comparisons to ortholog sequences from other asterids obtained from NCBI GenBank (e.g., Nerium, Coffea, Solanum). Various versions of the chloroplast genome sequence were used as a reference in alignreads to further refine the sequence based on new assemblies. Discrepancies between the assembly consensus sequences in introns and intergenic spacers were explored at the microread-level in Tablet to identify the correct sequence. An Asclepias tuberosa sequence [GenBank:GQ248251.1] served as a reference in alignreads to correctly assemble the $t r n H$ - psbA region.

In order to produce the highest quality $A$. syriaca chloroplast genome sequence and annotation, the consensus sequences were also aligned to chloroplast genome sequences for other individuals of $A$. syriaca produced in alignreads and Velvet 1.0.12 [39] based on either 80 bp single end or paired end Illumina sequences (S. Straub and A. Liston, unpublished data). Discrepancies between the consensus sequences from these assemblies and the original consensus sequences were resolved as either intraspecific variation, assembly or alignment errors in the $40 \mathrm{bp}$ read data set, or unresolved assembly errors. Informatically unresolved errors (e.g., AT-rich repeats) were corrected by obtaining Sanger sequences spanning those regions. Gene sequences that were expected to be divergent from the reference (e.g., $y c f 1$ ) and putative pseudogenes were also checked by Sanger sequencing. PCR reactions contained $\sim 10-20$ ng template DNA and had a final concentration of $1 \times$ Phusion ${ }^{\circledR}$ Flash High-Fidelity PCR Master Mix (Finnzymes) and $0.2 \mu \mathrm{M}$ of primer. PCR cycling conditions were $98^{\circ} \mathrm{C}$ for $30 \mathrm{~s}$ followed by 25 cycles of $98^{\circ} \mathrm{C}$ for 10 $\mathrm{s}$, primer set specific annealing temperature for $30 \mathrm{~s}, 72^{\circ}$ $\mathrm{C}$ for $30 \mathrm{~s}$, and a final extension of $72^{\circ} \mathrm{C}$ for $5 \mathrm{~min}$. See Table 1 for primer sequences and annealing temperatures. Cycling extension times were increased to $60 \mathrm{~s}$ and final extension times to $10 \mathrm{~min}$ for $a c c D$ and $c l p P$. Cycling extension times were increased to $90 \mathrm{~s}$ and final extension times to $10 \mathrm{~min}$ for $y c f 1-n d h F$ and rps15-ycf1. The success of PCR reactions was confirmed using agarose gel electrophoresis. The chloroplast and mitochondrial paralogs of $a c c D$ were co-amplified, bands excised from a $1.2 \%$ agarose gel, and purified using a QIAquick Gel Extraction Kit (Qiagen, Inc.). Due to low yield, the mitochondrial product was re-amplified using $1 \mu \mathrm{l}$ of extract as PCR template prior to sequencing. For additional chloroplast $a c c D$ sequencing, the mitochondrial product was virtually eliminated from the PCR pool by raising the annealing temperature to $61^{\circ} \mathrm{C}$. Sequences were produced using standard dye termination techniques by High-Throughput Sequencing Solutions at the University of Washington http://www.htseq.org or the Oregon State University CGRB. The quality of the finished $A$. syriaca chloroplast genome sequence was assessed by using it as the reference sequence in alignreads to re-assemble the $40 \mathrm{bp}$ reads and any discrepancies were explored and corrected.

The final annotation of the $A$. syriaca chloroplast genome was accomplished using BioEdit 7.0.5.3 [40] and visualized using GenomeVx [41]. The repeat content of the chloroplast genome was determined using the NCBI BLAST tool blast2seq. Only repeat motifs of greater than 30 bp and $85 \%$ sequence identity were characterized. Sequence identities were calculated in BioEdit on sequence alignments produced in MAFFT with the gapped positions removed. $\mathrm{Ka} / \mathrm{Ks}$ values for pseudogenes were calculated using the Bergen Center for Computational Science $\mathrm{Ka} / \mathrm{Ks}$ calculation tool http://services. cbu.uib.no/tools/kaks.

\section{Mitochondrial Genome}

The mitochondrial genome of $A$. syriaca was assembled using the alignreads pipeline and the $430,597 \mathrm{bp}$ mitochondrial genome sequence of Nicotiana tabacum 
Table 1 Primers used for Sanger sequence finishing of the Asclepias syriaca chloroplast genome

\begin{tabular}{|c|c|c|c|}
\hline Region spanned & Orientation & Primer Sequence $\left(5^{\prime}-3^{\prime}\right)$ & Annealing Temp. $\left({ }^{\circ} \mathrm{C}\right)$ \\
\hline \multirow[t]{2}{*}{$n d h C-t r n V$} & FORWARD & GCTCGATTCGAATTGTCAAGTCATCC & 57 \\
\hline & REVERSE & AGGCCCTCCGATAGGATACTCAAA & \\
\hline \multirow[t]{2}{*}{$\operatorname{trn} Q-p s b K$} & FORWARD & AAACCCGTTGCCTTACCACTTG & 57 \\
\hline & REVERSE & CACGGGTATGACTGGCATAACATC & \\
\hline \multirow[t]{2}{*}{$r b c L-a c c D$} & FORWARD & ATCCGTGAGGCTAGCAAATGGAGT & 59 \\
\hline & REVERSE & AGGCCCTAGTCCACCAATATGGAA & \\
\hline \multirow[t]{8}{*}{$\overline{a c c D}$} & FORWARD & TAATAAACAATGCTAGCGATTGGG & 57,61 \\
\hline & REVERSE & TACAATTAGATCTTAGGTCGCCAA & \\
\hline & FORWARD & GAACCTACACGCAAGCTAGTG & \\
\hline & FORWARD & TAAATGGCATTCCCATAGCAGT & \\
\hline & FORWARD & TGATATCGATGCGCAAGCTAG & \\
\hline & REVERSE & CCTATAAATGTAACTAGCACTGCA & \\
\hline & REVERSE & GGTAGCGTATTCAATCAAACGG & \\
\hline & REVERSE & CCAAGTATCCGGATCGATCGA & \\
\hline \multirow[t]{8}{*}{$c l p P$} & FORWARD & AATTGTATACATAATGGGCTGGT & 57 \\
\hline & REVERSE & TATTTATTCATTGCCCTGTTCGT & \\
\hline & FORWARD & AACTACTATGATGGCTCCGTTG & \\
\hline & FORWARD & CATAAGACCCATAATTTGATTTGAG & \\
\hline & FORWARD & GTTATGCTGTACTCCGGGTA & \\
\hline & REVERSE & GGTCAATTITATTGTAAAGCCGTAT & \\
\hline & REVERSE & TATGCAATTTATAAAACCAGATGTCC & \\
\hline & REVERSE & GTGAATTGAAAAAGTAGAACGTCAT & \\
\hline \multirow[t]{2}{*}{ rps8 -rp/14 } & FORWARD & CTCAGCAATAGTGTCTCTGCCCAT & 57 \\
\hline & REVERSE & AGAGCTGTAATTGTGCGTACCCGT & \\
\hline \multirow[t]{4}{*}{ ycf1-ndhF } & FORWARD & TCCATCCATATCCCAATTCCATTCA & 55 \\
\hline & REVERSE & GGGCATTAGAGGATTGGCTAAAGT & \\
\hline & FORWARD & ACGCCTCTGCATCTAGTATTG & \\
\hline & REVERSE & GGATCTIITGTCGAGAGCTTC & \\
\hline \multirow[t]{2}{*}{ ndhG-ndhl } & FORWARD & ATGCGGGCGGGTTGATATATGGTT & 59 \\
\hline & REVERSE & ATTGCTTTGGGTCGGTTACCAACGTC & \\
\hline \multirow[t]{4}{*}{ rps15-ycf1 } & FORWARD & GGCCTAATTGATACCTCAGCGGTT & 58 \\
\hline & REVERSE & TTATCAACGATGGAACCGCCCACT & \\
\hline & FORWARD & TATGTCTCGGTCTAGGAGAGG & \\
\hline & REVERSE & TGTACAAGTTGATTCAGGCGA & \\
\hline
\end{tabular}

For each region, additional primers appearing after the two original PCR primers are internal sequencing primers.

[GenBank:BA000042.1] [42] as a reference. Mitofy [43] was used to characterize the complement of protein coding, rRNA, and tRNA genes in the resulting contigs. The annotation of the mitochondrial genome of $N$. tabacum was updated using Mitofy before comparison of the gene content of the two genomes. BLAT [44] under default parameters was used to estimate the percentage of each $A$. syriaca mitochondrial gene assembled by calculating the proportion of the total length of the $N$. tabacum mitochondrial genes with hits in the A. syriaca contigs. The presence or absence of the cox1 group I intron in Asclepias was determined by using the Hoya sikkimensis (Apocynaceae) cox1 sequence [GenBank:AJ247588.1] as a BLAT reference.

\section{Ribosomal DNA}

The nuclear rDNA cistron, including 18S, 5.8S, and 26S, was assembled in alignreads using a $669 \mathrm{bp}$ partial sequence from A. syriaca [GenBank:AM396892] spanning the internal transcribed spacers (ITS) as the reference. Following the initial assembly, the assembly was repeated using the reverse complement of the reference sequence as the new reference to correct for an inconsistency in YASRA that caused the assembly result to be strand specific. A consensus sequence of the contigs obtained through the first two analyses was then used as the reference for a final assembly. A $5 \mathrm{~S}$ rDNA sequence for A. syriaca was assembled using a $330 \mathrm{bp}$ sequence including the $5 \mathrm{~S}$ locus from Solanum iopetalum 
[GenBank:AJ226045] as a reference in both the forward and reverse directions.

To assess intragenomic polymorphism, the complete genomic read pool was filtered for reads matching the consensus sequences of the assembled rDNA loci using BLAT and custom scripts [45]. Reads were discarded if their average Phred score was less than 20, and the remaining reads filtered so that any base in a read with a Phred score less than 20 was converted to ' $N$ ' using the FASTX-Toolkit [46]. Modified reads were assembled against the reference sequence using BWA [47], and at each position the number of reads differing from the consensus was tallied and a proportion calculated (script available from $\mathrm{KW})$. In order to estimate the error introduced by the sequencing method, the same procedure was applied to the PhiX174 control lane of the sequencing run.

Positions with $2 \%$ or more of reads differing from the consensus were considered to represent true polymorphism. This cutoff is comparable to error rates previously found using a similar quality-filtering scheme [48], but may be somewhat conservative as it is much higher than the error seen in the PhiX lane. See additional file 1: Polymorphism detected among Illumina reads for the PhiX sequencing control. All positions of the PhiX genome exhibited $<0.7 \%$ differing reads except position 3132 , which had $>17 \%$ of reads differing from the consensus. While the reason for this anomaly is uncertain, the overall average error rate in the PhiX lane is $<0.05 \%$.

\section{De novo assembly}

Data Quality Control Perl scripts were used to remove microreads containing N's and those reads corresponding to Illumina adapter sequences from the chastity-filtered short read pool to create a cleaned read pool [45]. The remaining reads were trimmed of bases of low quality or unscorable quality (quality score "B" in Illumina fastq files) and all bases subsequent to these bases to produce a cleaned and trimmed read pool [45].

De novo assembly of the cleaned read pool was performed using VelvetOptimiser 2.1.7 [49] and Velvet 1.0.12 with the minimum contig length set to $100 \mathrm{bp}$. Hash lengths between 19 and 31 were screened to find the optimal hash length for maximizing the total bases included in contigs. The expected coverage and the optimum coverage cutoff were estimated by VelvetOptimiser to be 2 and 0.3777216 respectively. This analysis was repeated for the cleaned and trimmed read pool. Use of the cleaned read pool resulted in more total bases in contigs; therefore this pool was used for all downstream de novo analyses. In order to further characterize the $A$. syriaca nuclear genome, BLAT was used to remove reads corresponding to nuclear rDNA and the chloroplast and mitochondrial genomes using the sequences generated through reference guided assembly. The de novo assembly was repeated to produce the set of contigs used for the non-rDNA repetitive element categorization and microsatellite marker development. The organellar genome and rDNA BLAT analyses were also used to estimate the percentage of overall reads corresponding to the high copy fraction of the genome.

Repetitive Element Characterization Non-ribosomal nuclear repetitive elements were characterized by submitting the de novo contigs to the MAKER [50] web annotation service http://www.yandell-lab.org/software/ mwas.html and categorizing the RepeatMasker [51] output from the MAKER run. The percentage of unique read hits to these contigs was determined using BLAT, with minimum sequence identity set to $70 \%$, to find hits to the contigs of putative DNA transposon or retrotransposon origin. The overall percentage of reads corresponding to known repetitive elements was calculated using BLAT (minimum sequence identity $=70 \%$ ) to determine the number of reads that hit the Viridiplantae repeat sequences downloaded from the Repbase Update release 15.07 database [52].

Microsatellite Marker Development De novo contigs of at least $150 \mathrm{bp}$, to allow for sufficient flanking sequence for primer design, were scanned for microsatellites using BatchPrimer3 [53]. Primers were designed flanking perfect di- and trinucleotide repeats of four or more repeat units and perfect tetra-, penta-, and hexanucleotide repeats of three or more repeat units such that the PCR amplicon sizes ranged between 100 and $450 \mathrm{bp}$ and primers met the default criteria for SSRs primers (Table 2; also see additional file 2: Primer sets designed using BatchPrimer3 for 184 nuclear microsatellite loci in Asclepias syriaca not tested for amplification success). Contigs containing at least one microsatellite locus for which primers could be designed were submitted to the PLAN web service [54], with the 'DNA to DNA: blastn against NCBI NT 20081206 database, top 10 hits with e $<=0.1$ cut-off' option selected, in order to check if any chloroplast or mitochondrial sequences had not been eliminated from the read pool used to generate the de novo contigs. To produce the best set of primers for candidate nuclear microsatellite locus development, those primer sets originating from contigs with a chloroplast or mitochondrial BLAST [55] hit with an E-value of $1 \mathrm{e}^{-5}$ or lower were removed from the candidate pool.

Primers were tested for amplification success for the 25 top candidate loci, which were selected for having the highest number of repeat units among all candidates. PCR amplification was conducted on genomic DNA of the same $A$. syriaca accession used for genome sequencing in $50 \mu \mathrm{l}$ reactions. Each reaction consisted of $1 \mu \mathrm{l}$ DNA extract template, $1.25 \mathrm{U} \mathrm{GoTaq}{ }^{\circledR}$ Flexi DNA Polymerase and $1 \times$ Green Flexi Reaction Buffer 
Table 2 Primers, PCR reaction conditions, and characteristics of microsatellite loci tested for amplification success in Asclepias syriaca

\begin{tabular}{|c|c|c|c|c|c|}
\hline Primer Set Name & Orientation & Primer Sequence $\left(5^{\prime}-3^{\prime}\right)$ & Annealing Temp. $\left({ }^{\circ} \mathrm{C}\right)$ & Repeat Motif & Product Size (bp) \\
\hline \multirow[t]{2}{*}{ As104467 } & FORWARD & ACTIITTGTTCTGTTCCGAGT & 52.9 & $\mathrm{TA}_{6}$ & 100 \\
\hline & REVERSE & CGGGGCTAGTATAGAAGAAGA & & & \\
\hline \multirow[t]{2}{*}{ As106994 } & FORWARD & AGGAAGCCAATTCAAGTAAAG & 48.9 & $\mathrm{GA}_{5}$ & 129 \\
\hline & REVERSE & GAAAATCCTTGCATGAAACT & & & \\
\hline \multirow[t]{2}{*}{ As12122 } & FORWARD & TTGGTTGTTGGAACTTACACT & 50.4 & $\mathrm{ATTC}_{4}$ & 115 \\
\hline & REVERSE & AAGCACAAGAATCAGAACAAT & & & \\
\hline \multirow[t]{2}{*}{ As125636 } & FORWARD & GAACTCCTATGATCTITCATIT & 50.8 & $\mathrm{TC}_{4} \ldots \mathrm{TA}_{4}{ }^{*}$ & 136 \\
\hline & REVERSE & AGACATCATGAACCCTCTITA & & & \\
\hline \multirow[t]{2}{*}{ As12641 } & FORWARD & СTCTTCATCTGGTTTCTCTTG & 49.0 & $\mathrm{TA}_{4}$ & 130 \\
\hline & REVERSE & TGCATACAAATTTAGGTGATTC & & & \\
\hline \multirow[t]{2}{*}{ As13110 } & FORWARD & AACCATAAATGCAGGTTCTIT & 50.9 & $\mathrm{TA}_{4}$ & 101 \\
\hline & REVERSE & GCATATCTAGCTCGCATTTAG & & & \\
\hline \multirow[t]{2}{*}{ As16135 } & FORWARD & TTAGGTTGTGAGAAGGGATTT & 50.3 & $\mathrm{CCT}_{4}$ & 100 \\
\hline & REVERSE & GCTITATTAATGTGGGAGGA & & & \\
\hline \multirow[t]{2}{*}{ As16369 } & FORWARD & AACGCTTATCCCTCACTITAC & 51.5 & $\mathrm{TA}_{5}$ & 100 \\
\hline & REVERSE & CCGAACTCTATATGAGTCCAAT & & & \\
\hline \multirow[t]{2}{*}{ As196582 } & FORWARD & GCATTATTGCATTGTCAAA & 50.5 & $\mathrm{TGA}_{5}$ & 113 \\
\hline & REVERSE & GCCGTTGTTCCTCTATAATTT & & & \\
\hline \multirow[t]{2}{*}{ As2003a } & FORWARD & CCCTCGTTATTGAGAAAATAGA & 50.5 & $\mathrm{ATA}_{5}$ & 105 \\
\hline & REVERSE & GGCTCTTACCAAAGAAAAGAT & & & \\
\hline \multirow[t]{2}{*}{ As20679a } & FORWARD & GTCATCGGAAGCTCTITTC & 52.7 & $\mathrm{CT}_{4}$ & 104 \\
\hline & REVERSE & AGTCAAAAGTCACACTCATCTG & & & \\
\hline \multirow[t]{2}{*}{ As2338 } & FORWARD & CATCTTGGTTAGCTCGTAAAC & 51.7 & $\mathrm{TC}_{5}$ & 104 \\
\hline & REVERSE & CCTTACCTAGGATGAAAGGAG & & & \\
\hline \multirow[t]{2}{*}{ As23491a } & FORWARD & ATTTCCTCCATTTGTTCTTTC & 50.0 & $\mathrm{TC}_{5}$ & 101 \\
\hline & REVERSE & GAAGTITGTCTGAAATGGTTG & & & \\
\hline \multirow[t]{2}{*}{ As2366 } & FORWARD & TTGGTGTTGTAAAGTGTTCCT & 54.5 & $\mathrm{TA}_{5}$ & 101 \\
\hline & REVERSE & ACCAGCTCGTCAGTCAAG & & & \\
\hline \multirow[t]{2}{*}{ As23942 } & FORWARD & GTGATTCCATTTACCATACC & 51.7 & $\mathrm{AG}_{5}$ & 100 \\
\hline & REVERSE & ATCCTTAGCAAATAGGAGTCG & & & \\
\hline \multirow[t]{2}{*}{ As23961 } & FORWARD & ACTCTTGTTATTTTGGGGAAT & 49.3 & $\mathrm{TG}_{5}$ & 108 \\
\hline & REVERSE & CAAAAGCTTTACACGATCAAT & & & \\
\hline \multirow[t]{2}{*}{ As282930 } & FORWARD & AACTTAGCTTTGGATGATGTTT & 50.1 & $\mathrm{TAA}_{4}$ & 102 \\
\hline & REVERSE & CAATTGGGCTTGTATATCAGA & & & \\
\hline \multirow[t]{2}{*}{ As28460 } & FORWARD & CACAAGATGAACATCAAAACC & 49.6 & $\mathrm{AT}_{5}$ & 100 \\
\hline & REVERSE & TTGAGTATATTTGTTCCCCAAT & & & \\
\hline \multirow[t]{2}{*}{ As292191 } & FORWARD & GGAGGGTCTAATGTCTGATGT & 51.3 & $\mathrm{TG}_{5}$ & 125 \\
\hline & REVERSE & ATTCACACATCCCATACCAT & & & \\
\hline \multirow[t]{2}{*}{ As43163 } & FORWARD & TATTCCAAGAAATTGGTCAAC & 49.7 & $\pi \mathrm{A}_{4}$ & 110 \\
\hline & REVERSE & CATGGAAGAAGAACAAAACAG & & & \\
\hline \multirow[t]{2}{*}{ As5768 } & FORWARD & AAGGGAAATGATCCTTAGGTA & 51.0 & $\mathrm{AG}_{6}$ & 108 \\
\hline & REVERSE & TTCGAAAGAGTTCCACATCTA & & & \\
\hline As71725 & FORWARD & TTTACGACTCATGTACTCTTCC & 50.8 & $\mathrm{AG}_{4} \ldots \mathrm{TG}_{5}{ }^{*}$ & 133 \\
\hline & REVERSE & GTAATACCGGAAAATGACCTC & & & \\
\hline As77693 & FORWARD & GTCAGTCAACTCAACAATGCT & 51.0 & $\mathrm{TC}_{6}$ & 102 \\
\hline & REVERSE & TCCCAAAACTCAGATCTAACA & & & \\
\hline As81784 & FORWARD & AATGTAAAGGGAGATGTTTATCA & 52.0 & $\mathrm{TAT}_{4}$ & 129 \\
\hline & REVERSE & AGCATCAAGAACTTGAACAGA & & & \\
\hline As99212 & FORWARD & TCTATGGTCAACAATTTCTGC & 53.4 & $\mathrm{AT}_{5}$ & 153 \\
\hline & REVERSE & TITATAGGGGTAGCGTAGGTG & & & \\
\hline
\end{tabular}


(Promega, Madison, WI, USA), $2.5 \mathrm{mM} \mathrm{MgCl}_{2}, 200 \mu \mathrm{M}$ dNTPs, and $0.8 \mathrm{mM}$ amplification primers. PCR was carried out in a $\mathrm{C} 1000^{\mathrm{TM}}$ thermal cycler (BioRad, Hercules, CA, USA) under the following conditions: initial denaturing at $95^{\circ} \mathrm{C}$ for $2 \mathrm{~min}, 35$ cycles of $95^{\circ} \mathrm{C}$ for 30 sec-variable annealing temperature for $30 \mathrm{sec}-72^{\circ} \mathrm{C}$ for 1 min, followed by a final extension at $72^{\circ} \mathrm{C}$ for $5 \mathrm{~min}$. See Table 2 for annealing temperatures. Amplicons of the predicted size were confirmed using agarose gel electrophoresis.

\section{Nuclear genome characterization and development of low copy nuclear markers}

In order to provide an initial characterization of nuclear gene content and coverage, all 19,899 available ESTs for Catharanthus roseus were downloaded from GenBank on 29 November 2010. See additional file 3: List of GI numbers for 19,899 Catharanthus roseus ESTs downloaded from GenBank and assembled into unigenes. The sequences were subjected to simple cleaning in SeqClean (Dana Farber Cancer Institute; available at http:// sourceforge.net/projects/seqclean). Cleaned and trimmed sequences were assembled into unigenes using iAssembler v1.2 (available at http://bioinfo.bti.cornell.edu/tool/ iAssembler). Unigenes with BLAT hits corresponding to the rDNA or chloroplast or mitochondrial genomes of A. syriaca were excluded from this set. Simple repeats, low complexity sequences, and long terminal repeats were masked in the remaining unigenes using the RepeatMasker web service [[51]; available at http://www. repeatmasker.org/cgi-bin/WEBRepeatMasker]. BLAT with a tile size of 7 and minimum sequence identity of $80 \%$ was used to find read hits to the remaining putatively nuclear masked unigenes. The number of unique microread hits per unigene was used to calculate the hits per $\mathrm{kb}$ of sequence based on the length of gene. Coverage for these genes was then estimated using the formula [(hits per $\mathrm{kb}$ of sequence $\times$ length of the microread)/1000]. Coverage is overestimated when reads overlap. JMP 9.0.0 (SAS Institute, Inc.) was used to calculate coverage distributions. A Monte Carlo resampler implemented in PopTools [56] was used to calculate 95\% bootstrap confidence intervals (1,000 iterations) for median hits per $\mathrm{kb}$ and coverage values.

With low genome coverage, few if any single-copy nuclear genes were expected to have been assembled in de novo contigs. In order to extract useful information for nuclear marker development from the unassembled, uncleaned pool of reads and further explore the $A$. syriaca gene space, BLAT with a tile size of 7 and minimum sequence identity of $80 \%$ was used to find reads that hit alignments for 1,086 single-copy orthologs from other asterids [e.g., tomato (Solanum lycopersicum), potato (Solanum tuberosum), pepper (Capsicum annuum), coffee (Coffea canephora)] and Arabidopsis thaliana [COS II markers; [57]]. The average length of sequences included in each alignment was used as the gene length in subsequent calculations, which were completed as described above for the Catharanthus unigene set.

The COSII pool of candidate genes was most appropriate for the development of markers intended for phylogenetics because single-copy genes are preferred in order to avoid ortholog/paralog conflation. MAFFT 6.240 was used to align the Asclepias microreads with the COSII alignments using the localpair option and 1,000 cycles of iterative refinement. Primers were designed in exons flanking the putative locations of one or more introns based on the location of introns in $A$. thaliana indicated by the Sol Genomics Network's intron finder application http://solgenomics.net/tools/ intron_detection/find_introns.pl for genes with $0.5 \times$ or greater coverage (Table 3 ). If possible, primers were designed with GC clamps and were chosen from sections of the read identical to or highly similar to the coffee sequence without mismatches near the 3 ' end of the primer sequence.

The primers designed using the $A$. syriaca microreads were tested for amplification success and their utility in phylogenetic studies of Asclepias and across Apocynaceae. Vouchers for all DNA samples used are listed in Table 4. DNA was extracted as described above for $A$. syriaca. PCR amplification was conducted in $50 \mu \mathrm{l}$ reactions on genomic DNA of the same A. syriaca accession used for genome sequencing, 16 accessions of 13 other species of American Asclepias, including four accessions of two morphotypes of A. pringlei, one species of Gomphocarpus belonging to the sister clade to American Asclepias, and one sample each of successive sister groups to the clade including Asclepias and Gomphocarpus (Calotropis and Pergularia). Each reaction consisted of $1 \mu \mathrm{l}$ template DNA extract, $1.25 \mathrm{U} \mathrm{GoTaq}^{\circledR}$ Flexi DNA Polymerase and $1 \times$ Green Flexi Reaction Buffer (Promega, Madison, WI, USA), $2.5 \mathrm{mM} \mathrm{MgCl}_{2}, 200 \mu \mathrm{M}$ dNTPs, and $0.8 \mathrm{mM}$ amplification primers. PCR was carried out in a $\mathrm{C} 1000^{\mathrm{TM}}$ thermal cycler (BioRad, Hercules, CA, USA) under the following conditions: initial denaturing at $94^{\circ} \mathrm{C}$ for $2 \mathrm{~min}, 35$ cycles of $94^{\circ} \mathrm{C}$ for $30 \mathrm{sec}$-variable annealing temperature for $30 \mathrm{sec}-72^{\circ} \mathrm{C}$ for $1 \mathrm{~min}$, followed by a final extension at $72^{\circ} \mathrm{C}$ for $5 \mathrm{~min}$. See Table 3 for primer sequences and annealing temperatures.

For the Apocynaceae-wide analyses, species from four tribes (Marsdenieae, Baisseeae, Apocyneae, Alyxieae) at increasing phylogenetic distance from Asclepias [58] were selected for primer testing. Pairs of species from three tribes were used: Telosma cordata and Marsdenia glabra (Marsdenieae), Baissea multiflora and Oncinotis tenuiloba (Baisseeae), Epigynum auritum and E. 
Table 3 Primers designed from Asclepias syriaca microreads hitting COSII alignments

\begin{tabular}{|c|c|c|c|}
\hline Primer Set Name & Orientation & Primer Sequence $\left(5^{\prime}-3^{\prime}\right)$ & Annealing Temp. $\left({ }^{\circ} \mathrm{C}\right)$ \\
\hline \multirow[t]{2}{*}{ At1g24360a } & FORWARD & GCAAGATCATCAAAGGAGGCA & 59.0 \\
\hline & REVERSE & GATTTAGATCAATAACCTCCTGCCA & \\
\hline \multirow[t]{2}{*}{ At1g24360b } & FORWARD & GGAGGTTATTGATCTAAATCTCAC & 56.3 \\
\hline & REVERSE & AACAAGGCCAACAACTGATG & \\
\hline \multirow[t]{2}{*}{ At1g24360c } & FORWARD & CAATTACAGTGCTGCAAAAGCTG & 60.0 \\
\hline & REVERSE & CATATCAGATGCAATGAATCCGGG & \\
\hline \multirow[t]{2}{*}{ At1g44760a } & FORWARD & TACTCATGTTACTAATAAGGGTGA & - \\
\hline & REVERSE & CTTTGCTTGCTTCCTCACTC & \\
\hline \multirow[t]{2}{*}{ At1g55880a } & FORWARD & ATGTACACAAAAGAGGAGGCTG & 54.0 \\
\hline & REVERSE & GATGCCTCATCCCACTATCAC & \\
\hline \multirow[t]{2}{*}{ At1g77370a } & FORWARD & CCAAAGCGCCATCTACTCCA & 60.0 \\
\hline & REVERSE & GGCCAACCAAATCCAGAAGAAT & \\
\hline \multirow[t]{2}{*}{ At2g03120a } & FORWARD & GAAGGTAGATCCAAATTTGAATGTC & 58.0 \\
\hline & REVERSE & GCAAGTCAACACAGCATTAAC & \\
\hline \multirow[t]{2}{*}{ At2g06530a } & FORWARD & GTGAAGGTAATGGCCAAAGATC & 58.0 \\
\hline & REVERSE & GTAACACCTTTCATTGCTTCTCC & \\
\hline \multirow[t]{2}{*}{ At2g21960a } & FORWARD & ATTGGGGTTTCTTTGTTCCATAC & 53.0 \\
\hline & REVERSE & GCAGATTGGCCAACAACTTTG & \\
\hline \multirow[t]{2}{*}{ At2g21960b } & FORWARD & GTTGTTGGCCAATCTGCAGA & 59.0 \\
\hline & REVERSE & TITGTTGCTGATCTTTGCTAAGC & \\
\hline \multirow[t]{2}{*}{ At2g30200a } & FORWARD & ACAAGCTGTTGGAATGGGTG & 55.0 \\
\hline & REVERSE & ATCTCAACTGCAGCTAAGCTG & \\
\hline \multirow[t]{2}{*}{ At2g30200b } & FORWARD & ATCTATGTCACCAGCTTAGCTG & 58.3 \\
\hline & REVERSE & TITGAATGATTTGCCTTTGCTTC & \\
\hline \multirow[t]{2}{*}{ At2g30200c } & FORWARD & CAAAGGCAAAATCATTCAAAGC & 56.3 \\
\hline & REVERSE & TGCATCAACATTTGATATTACTGG & \\
\hline \multirow[t]{2}{*}{ At2g34620a } & FORWARD & TTCAGAAAGGTCATCAACAAATG & 57.8 \\
\hline & REVERSE & GCTGAAAGTGAACAAACCTGG & \\
\hline \multirow[t]{2}{*}{ At2g34620b } & FORWARD & TGCCAACATTTCTITACAATTCAAG & 52.0 \\
\hline & REVERSE & none & \\
\hline \multirow[t]{2}{*}{ At2g47390a } & FORWARD & GTCTTGGTACAAAACACGCAAG & 59.0 \\
\hline & REVERSE & TCAGTITAGATTCTTTAGAGGTAAG & \\
\hline \multirow[t]{2}{*}{ At2g47390b } & FORWARD & CTAATGAGTTTGCTGGCATTGG & - \\
\hline & REVERSE & CACCATGTCCTITGAGTGCG & \\
\hline \multirow[t]{2}{*}{ At4g13430a } & FORWARD & GAGCAAAACATAAAGTACTTCTA & 48.0 \\
\hline & REVERSE & CATTTCACCATCCATAACAA & \\
\hline \multirow[t]{2}{*}{ At4g13430b } & FORWARD & GTGCCTCCAACATTGAGATTTG & 59.0 \\
\hline & REVERSE & CATTCTCTAGCCAAAGCACGA & \\
\hline \multirow[t]{2}{*}{ At $4 \mathrm{~g} 13430 \mathrm{c}$} & FORWARD & GTGCTITGGCTAGAGAATGCA & 60.0 \\
\hline & REVERSE & CAAGACAAGCACCACAACTAGG & \\
\hline \multirow[t]{2}{*}{ At4g26750a } & FORWARD & GCCTGATGACCATTTACATCTTG & - \\
\hline & REVERSE & TACAGGTCTCCTCCCTTCTITC & \\
\hline \multirow[t]{2}{*}{ At5g13420a } & FORWARD & TGGTATGACAACCTGTGCCG & 55.0 \\
\hline & REVERSE & GTATCATCAGCAAGTCTTGGTGAA & \\
\hline At5g13420b & FORWARD & CTCCATGCGTCCCTTCAATC & 60.5 \\
\hline & REVERSE & TGGCACCTTTCTTCACCAGAG & \\
\hline At5g23540a & FORWARD & GTGTTGAAGCGGTTGATCATG & - \\
\hline & REVERSE & TCCATCTGTCCATTTTTTCTTATGA & \\
\hline At5g23540b & FORWARD & none & 52.0 \\
\hline
\end{tabular}


Table 3 Primers designed from Asclepias syriaca microreads hitting COSIl alignments (Continued)

\begin{tabular}{llll}
\hline & REVERSE & AAGGCATCAATTACCACTTTCC & \\
\hline At5g49970a & FORWARD & GTAGCTGCTCGTCATTATATC & 55.8 \\
& REVERSE & GAGAATCCAAACATTGCATCA & 57.8 \\
\hline At5g49970b & FORWARD & TTGATGCAATGTTGGATTCTC & \\
& REVERSE & GGCACACAATTTTGGAGCAG & \\
\hline
\end{tabular}

Multiple primer sets were designed for loci where multiple read hits allowed potential amplification of different introns. Primer set names correspond to the orthologs of these genes in Arabidopsis. Listed annealing temperatures were used in amplifications of Asclepias samples. An annealing temperature of $55^{\circ} \mathrm{C}$ was used for all loci for amplifications of other Apocynaceae samples.

cochinchinense (Apocyneae); Oncinotis glabrata was substituted for $O$. tenuiloba for six of the primer pairs. In addition, the even more distantly related Alyxia oblongata and, for six of the primer pairs, Al. grandis (Alyxieae) were tested. DNA was extracted using a modified DNeasy method [58]. PCR reactions were performed in $30 \mu \mathrm{l}$ reaction volume with Apex ${ }^{\mathrm{TM}}$ Taq DNA Polymerase Master Mix (Genesee Scientific) diluted to $1 \times$ with final concentrations of $1.5 \mathrm{mM} \mathrm{MgCl} 2$ and $0.5 \mathrm{mM}$ forward and reverse primers (Table 3). One $\mu$ l of unquantified DNA extract was added to each reaction. PCR was performed in an Eppendorf Mastercycler gradient thermocycler with an initial melting step of $94^{\circ} \mathrm{C}$ for 3 min, followed by 35 cycles of $94^{\circ} \mathrm{C}$ for $1 \mathrm{~min}, 55^{\circ} \mathrm{C}$ for 1 min, $72^{\circ} \mathrm{C}$ for $1 \mathrm{~min}$, with a final extension of $72^{\circ} \mathrm{C}$ for 7 min. PCR products were examined for amplification success and product sizes estimated by agarose gel electrophoresis for both the Asclepias-specific and familywide surveys.

\section{Results \& Discussion}

Genome size estimation

The average $2 \mathrm{C}$ value for the five $A$. syriaca individuals was $1.68 \mathrm{pg}$ (SD 0.07), which corresponds to a haploid

Table 4 Herbarium voucher specimens for DNA samples included in the COSII marker screen

\begin{tabular}{|c|c|c|}
\hline Species & Voucher & Provenance \\
\hline Alyxia grandis P.I. Forst. & D. J. Middleton 703 [A] & Australia \\
\hline Alyxia oblongata Domin. & D. J. Middleton $702[\mathrm{~A}]$ & Australia \\
\hline Asclepias californica Greene ssp. californica & Lynch 10,779 [LSUS] & USA. California. San Bernardino Co. \\
\hline Asclepias connivens Baldwin ex Elliott & Lynch 12,350 [LSUS] & USA. Florida \\
\hline Asclepias coulteri A. Gray & Fishbein 5172 [OKLA] & Mexico. Querértaro. Mpio. Landa \\
\hline Asclepias cryptoceras S. Watson ssp. davisii & Fishbein 5723 [OKLA] & USA. Oregon. Crook Co. \\
\hline Asclepias fascicularis Decne. & Fishbein 5886 [OKLA] & USA. California. Siskiyou Co. \\
\hline Asclepias glaucescens Kunth & Lynch 14,142 [LSUS] & Mexico. Oaxaca \\
\hline Asclepias oenotheroides Schltdl. \& Cham. & Lynch 13,339 [LSUS] & USA. Texas \\
\hline Asclepias otarioides E. Fourn. & Fishbein 5857 [OKLA] & Mexico. Distrito Federal. Del. Tlalpan \\
\hline Asclepias pringlei (Greenm.) Woodson & Fishbein 5845 [OKLA] & Mexico. Zacatecas. Mpio. Tlaltenango \\
\hline Asclepias pringlei & Ventura 2807 [ARIZ] & Mexico. Distrito Federal. Del. Milpa Alta \\
\hline Asclepias aff. pringlei & Fishbein 5119 [OKLA] & Mexico. Michoacán. Mpio. Coalcomán \\
\hline Asclepias aff. pringlei & Fishbein 5175 [OKLA] & Mexico. Querétaro. Mpio. Pinal de Amoles \\
\hline Asclepias sanjuanensis K.D. Heil, J.M. Porter \& S.L. Welsh & Ellison s.n. [OKLA] & USA. New Mexico. San Juan Co. \\
\hline Asclepias stenophylla A. Gray & Fishbein 5394 [OKLA] & USA. Colorado. Boulder Co. \\
\hline Asclepias subulata Decne. & Fishbein 3147 [WS] & Baja California Sur. Mpio. Comondú \\
\hline Asclepias viridis Walter & Lynch 13,072 [LSUS] & USA. Texas \\
\hline Baissea multiflora A. DC. & F. Billiet S3853 [BR] & Cultivated. Belgium. Nat. Bot. Gard. Belgium \\
\hline Calotropis procera (Aiton) W.T. Aiton & Fishbein 5472 [OKLA] & Cultivated \\
\hline Epigynum auritum (C.K. Schneid.) Tsiang \& P.T. Li & D. J. Middleton et al. 1457 [A] & Thailand \\
\hline Epigynum cochinchinensis (Pierre) D.J. Middleton & D. J. Middleton 209 [A] & Thailand \\
\hline Gomphocarpus cancellatus (Burm. f.) Bruyns & Drewe $534[\mathrm{~K}]$ & South Africa \\
\hline Marsdenia glabra Constantin & D. J. Middleton et al. 1123 [A] & Thailand \\
\hline Oncinotis glabrata Stapf ex Hiern & C. C. H. Jongkind et al. 1315 [US] & Ghana \\
\hline Oncinotis tenuiloba Stapf & T. Abbott $7707[\mathrm{Z}]$ & South Africa \\
\hline Pergularia daemia (Forssk.) Chiov. & Fishbein 5445 [OKLA] & Namibia \\
\hline Telosma cordata (Burm. f.) Merr. & T. Livshultz 01-33 [BH] & Cultivated. USA. Cornell University \\
\hline
\end{tabular}


nuclear genome size of approximately $820 \mathrm{Mbp}$. Estimated 2C genome sizes in Apocynaceae range from 0.74 pg in Voacanga grandifolia to $5.00 \mathrm{pg}$ in tetraploid Ceropegia woodii [59], and correspond to haploid nuclear genome sizes of approximately $360-2,445 \mathrm{Mbp}$. Among the ca. 3,000 species of Asclepiadoideae [60], which include $C$. woodii, other $2 \mathrm{C}$ values for diploids are all greater than $4.30 \mathrm{pg}$ [59]. The genome of Asclepias is on the small side for the family based on current knowledge, but a comparison is difficult to make due to the limited sampling of genome size diversity in Apocynaceae.

\section{Illumina sequencing and quality filtering}

Of the 10,512,738 reads obtained, a total of 9,651,427 short reads passed the first step of quality filtering yielding $386 \mathrm{Mbp}$ of sequence. The raw reads were submitted to the NCBI Sequence Read Archive (SRP005621). Trimming reads of nucleotides with a "B" quality score and subsequent bases resulted in retention of $99.06 \%$ of the quality-filtered data. Reads yielding no data due to all unscorable bases accounted for $0.20 \%$ of this read pool. Of the reads obtained, approximately $11.8 \%, 3.4 \%$, and $1.8 \%$ originated from the chloroplast genome, mitochondrial genome, and rDNA repeat arrays respectively. Based on the estimated genome sizes, approximately $0.5 \times$ coverage, on average, of the whole $A$. syriaca genome (including organellar genomes) and $0.4 \times$ coverage of the nuclear genome were obtained from one lane of Illumina reads.

\section{Characterization of the organellar genomes of Asclepias syriaca \\ The Chloroplast Genome}

The chloroplast genome of $A$. syriaca is $158,598 \mathrm{bp}$ [GenBank:JF433943], excluding two small, unresolved regions that were not able to be assembled or Sanger sequenced due to polynucleotide stretches (rps8-rpl14 intergenic spacer and $\psi y c f 1$ ), and has an inverted repeat (IR) of 25,401 bp (Figure 1). The initial assembly produced using the alignreads pipeline and the oleander reference contained 51 contigs with a median read depth of $246 \times$ and N50 of 4,683 bp. The longest contig was $14,884 \mathrm{bp}$. The final assembly using the finished $A$. syriaca sequence as a reference contained 22 contigs, had an N50 of 9,030 bp, and longest contig of 28,186 bp. The use of chloroplast contigs from $80 \mathrm{bp}$ reads from other A. syriaca individuals (S. Straub and A. Liston, unpublished data) in combination with Sanger sequencing of select regions in the $0.5 \times$ genome individual and these other individuals, resulted in the addition of $6,622 \mathrm{bp}$ and deletion of 1,402 bp of sequence. That large insertions and deletions were found relative to the original assembly was expected due to the limited power of reference guided assembly algorithms to reconstruct these differences. Only 38 bp of sequence from the original reference guided assembly were determined to be incorrect, producing substitution errors in the genome sequence. The sum total of these changes resulted in the alteration of approximately $5 \%$ of the total sequence length, indicating that $95 \%$ of the chloroplast genome of A. syriaca was assembled at $0.5 \times$ average genome coverage with reads of only $40 \mathrm{bp}$ and an oleander reference with $85 \%$ sequence identity (considering only one copy of the inverted repeat). Although the original assembly was very good overall, comparison of the final assembly with the finished reference sequence highlighted the limitations of using 40 bp vs. 80 bp reads for sequence assembly. Even with a correct reference sequence, some regions were still not assembled properly (e.g., $a c c D$, $y c f 1$ ) and some assembly mistakes from the original assembly were recreated (e.g., an erroneous 5 bp deletion in the middle of $r b c L$ causing a frameshift).

In terms of overall size and IR size, $A$. syriaca has a typical asterid chloroplast genome [61]. The gene content of the A. syriaca chloroplast genome is similar to those of oleander and coffee (Coffea arabica) [62], with 110 unique genes (76 protein coding, 30 tRNA, 4 rRNA). Seventeen of these are fully duplicated in the IR for a total of 127 putatively functional genes. The most notable difference between the milkweed genome and the oleander and coffee chloroplast genomes is that $c l p P, a c c D$, and $y c f 1$ are likely pseudogenes in Asclepias. Another difference in the Asclepias genome relative to the other two genomes is shifting of the boundary of the IR in A. syriaca such that the truncated version of rps 19 observed in oleander and coffee is not present in IRa of milkweed. Assessment of smaller repeats showed that the A. syriaca chloroplast genome contains few repeats (Table 5). In comparison, the coffee chloroplast genome has nearly three times as many repeats of 30 or more basepairs with $90 \%$ or greater sequence identity [62].

The A. syriaca $\psi$ clpP has several features that indicate that it is likely a pseudogene. The nucleotide and protein sequences are divergent from those of oleander (81.3\% exon nucleotide and $67.0 \%$ protein sequence identity) and coffee (80.8\% exon nucleotide and 67.5\% protein sequence identity). In comparison, oleander and coffee have $97.2 \%$ exon nucleotide and $98.9 \%$ protein sequence identity. There is also a 28 bp insertion near the end of exon 3 of $A$. syriaca (validated by Sanger sequencing) that causes a frame shift and the introduction of a stop codon. Sequence comparisons showed that $\operatorname{clp} P$ in oleander and coffee are likely under negative selection $(\mathrm{Ka} / \mathrm{Ks}=0.07$ and 0.03 respectively); however, the $\mathrm{Ka} / \mathrm{Ks}$ for Asclepias is 1.15 indicating that this locus is likely evolving neutrally. While the $c l p P$ introns 


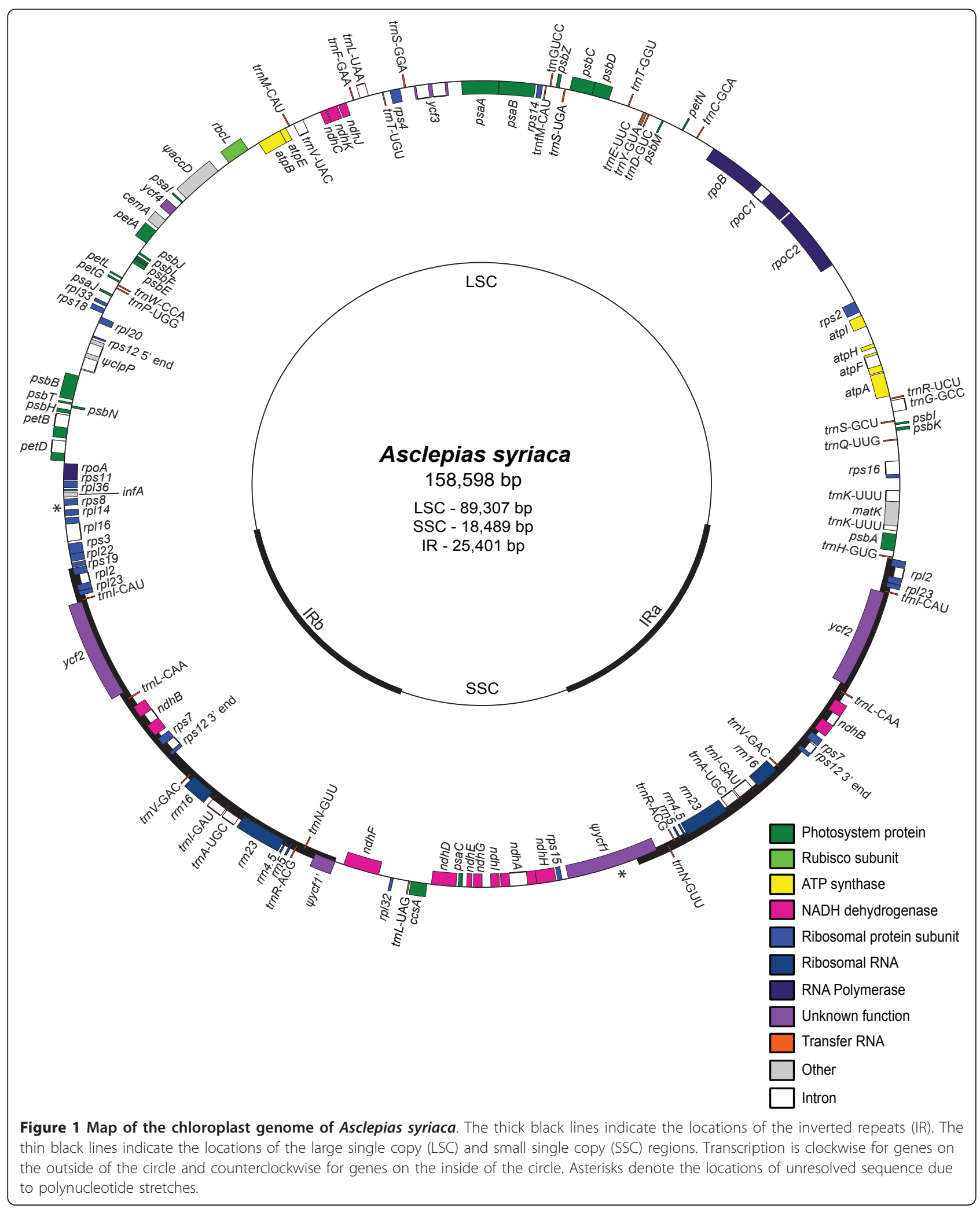


Table 5 Repeats in the Asclepias syriaca chloroplast genome

\begin{tabular}{|c|c|c|c|}
\hline Repeat Location(s) & Repeat Type & Repeat Length (bp) & Identity \\
\hline trnS-GCU:trnS-GGA & Inverted & 32 & $100 \%$ \\
\hline$\psi a c c D$ & Direct & 63 & $89 \%$ \\
\hline$\psi a c c D$ & Direct & 202 & $85 \%$ \\
\hline psbN-psbH spacer & Direct & 66 & $99 \%$ \\
\hline rpl23-trnl-CAU spacer & Direct & 57 & $97 \%$ \\
\hline $\operatorname{trnN-GUU-\psi yycf1~spacer~}$ & Direct & 299 & $87 \%$ \\
\hline
\end{tabular}

Repeats of at least 30 bp and at least $85 \%$ sequence identity to each copy are reported.

have been lost multiple times in angiosperms [e.g., $[63-65,67,68]]$, the coding region has been lost much less often. Other examples of loss of the coding region are only known from Scaevola (Goodeniaceae), Passiflora (Passifloraceae), Trachelium (Campanulaceae), Geranium and Monsonia (Geraniaceae) [67-69].

The $a c c D$ gene of $A$. syriaca is also likely a pseudogene. This gene is 2,584 bp in A. syriaca compared to $1,497 \mathrm{bp}$ in oleander. There is a repeat-rich (Table 5), approximately $1 \mathrm{~kb}$ insertion in the middle of the gene. Although the insertion does not interrupt the reading frame, the remainder of the sequence that is alignable with oleander is highly divergent. This gene can be highly variable, even within a genus [66], but due to the extent of sequence divergence, and lengths of the insertion and repeat motifs, this gene is likely non-functional in Asclepias. However, in pepper, a smaller, repeat-containing insertion of $144 \mathrm{bp}$ in $a c c D$ does not cause a frameshift nor prevent transcription of the gene [70]. There are numerous other examples of loss of $a c c D$ from the plastome among angiosperms [e.g., $[64,65,67-69,71]]$.

The assembly of $\psi a c c D$ proved especially challenging for Asclepias due to sequence divergence and the large insertion relative to the oleander reference. De novo assembly of 80 bp reads from another $A$. syriaca individual and Sanger sequencing confirmed the correct sequence. Further inspection indicated that the assembly errors in this region arose due to the presence of an $a c c D$ pseudogene in the mitochondrial genome of $A$. syriaca, also confirmed by Sanger sequencing, which was more similar in sequence and length to the oleander chloroplast $a c c D$. A similar problem involving $a c c D$ and a mitochondrial genome sequence was encountered in the assembly of the pepper plastome [70]. Problems of this sort are likely to arise for assembly of chloroplast genomes, especially with short reads, for regions of the genome that have been duplicated in the mitochondrial or nuclear genomes [72].

A third possible pseudogene in the A. syriaca chloroplast genome is $\psi y c f 1$. This gene contains one of the two polynucleotide repeats of unresolved length in the genome and has a highly divergent sequence compared to oleander and coffee. The sequence of this gene was also checked and improved using de novo contigs and Sanger sequencing to eliminate the possibility of assembly errors as a cause of sequence divergence. Although $y c f 1$ is one of the most quickly evolving chloroplast genes in seed plants [e.g., [66,73,74]], the full length copy of $y c f 1$ in Asclepias is so divergent that it may be non-functional. The sequence identity between $y c f 1$ in oleander and coffee is $87.5 \%$, whereas the sequence identities between each of those species and Asclepias are only $79.4 \%$ and $72.2 \%$ respectively. In addition to decreased sequence identity, there were 62 and 61 indels inferred from alignments of the Asclepias ycf1 sequence with the oleander and coffee sequences respectively, whereas there were only 16 indels inferred from an alignment of oleander and coffee. Among angiosperms $y c f 1$ has also been lost multiple times [e.g., [65,67-69,71]].

All three of the putative pseudogenes detected in the A. syriaca plastome, $\psi c l p P$, $\psi a c c D$, and $\psi y c f 1$ have functions that are known to be essential for plant development and survival [75-77]. Additional studies to evaluate the functionality of these putative pseudogenes will be needed to confirm their designation as such, in combination with further analysis of the nuclear genome to locate functional copies of these indispensible genes or determine which other nuclear genes might encode a functional replacement as in, for example, the case of $a c c D$ in grasses [e.g., [78,79]].

\section{The Mitochondrial Genome}

A total of $130,764 \mathrm{bp}$ of the A. syriaca mitochondrial genome was assembled in 115 contigs with a median read depth of 32x and N50 of 1,666 bp. See additional file 4: Asclepias syriaca mitochondrial genome contigs (also accessible at http://milkweedgenome.org). The longest contig was $6,948 \mathrm{bp}$. The extent to which the portion of the genome assembled for A. syriaca represents the whole mitochondrial genome is unknown due to the extensive variation observed for genome size, gene content, and organization among angiosperms $[43,80,81]$. Divergence from the organization of the Nicotiana reference likely prevented a more complete and contiguous assembly. Complete (rrn5) or nearly complete (rrn26, rrn18) ribosomal DNA sequences were identified among the contigs. Twenty-eight putative tRNA sequences representing 25 different tRNAs were also identified. Absence of a complete complement of tRNAs was not unexpected because the number of tRNAs in plant mitochondrial genomes is variable due to the replacement of native mitochondrial tRNAs with versions of chloroplast origin or import of nuclear encoded tRNAs of chloroplast or nuclear origin [80,81]. For example, among the tRNAs in the A. syriaca 
mitochondrial genome, both a mitochondrial version and a chloroplast version of trnE-TTC were detected.

The protein coding gene content observed for $A$. syriaca was compared to that of the mitochondrial genome of Nicotiana tabacum because it is the only other asterid with a sequenced mitochondrial genome. The assembled part of the genome contained the same 37 protein coding genes of known function as the $N$. tabacum mitochondrial genome with an average coverage of $94.8 \%$. Putative pseudogenes of $r p s 2, r p s 7$, and rps 14 were identified in the $A$. syriaca mitochondrial genome contigs. Both the mitochondrial genome of N. tabacum and A. syriaca appear to lack functional copies of these three genes, in addition to lacking rpl6, rps8, and rps11, the genes lost in all angiosperms, but present in Marchantia [42]. Evidence of urps 14 is present in tobacco, but there is no remaining evidence of the other two genes. Due to the pseudogenization or absence of these genes in the $N$. tabacum reference used for the A. syriaca assembly, a more complete characterization of the mitochondrial genome based on additional data will be required to determine if these genes are truly non-functional in $A$. syriaca or if their absence is an assembly artifact.

Extensive horizontal gene transfer has been hypothesized for the group I intron present in the $\operatorname{cox} 1$ gene of angiosperm mitochondrial genomes, with six horizontal transfer events hypothesized in Apocynaceae alone [82]. Alternatively, it has been argued that the distribution of the cox 1 intron in plants more likely involved a single horizontal transfer followed by intron loss in many lineages [83]. All but one of the Apocynaceae genera sampled have contained the cox 1 intron $[82,83]$, and it was also found to be present in the assembled $\operatorname{cox} 1$ gene of $A$. syriaca. This result adds to the evidence supporting the intron loss hypothesis over horizontal gene transfer in Apocynaceae.

\section{Characterization of the nuclear genome of Asclepias syriaca}

The Velvet de novo assembly using a hash length of 21 resulted in 1,399,320 bp of sequence in 6,997 contigs, $79 \%$ of which were 200 bp or less. See additional file 5: Asclepias syriaca nuclear genome de novo assembly contigs (also accessible at http://milkweedgenome.org). The longest contig was 5,027 bp and the N50 for the assembly was 196.

\section{Repetitive Element Characterization}

A total of 564 repetitive elements were detected in the A. syriaca de novo contigs (Figure 2). The majority of these repeats were Ty1/copia-like and Ty3/gypsy-like LTR retrotransposons (Figure 2), which are abundant in plants and common across eukaryotes [84-86]. Only the most highly represented repeats in the genome would have high enough coverage at $0.4 \times$ total nuclear genome

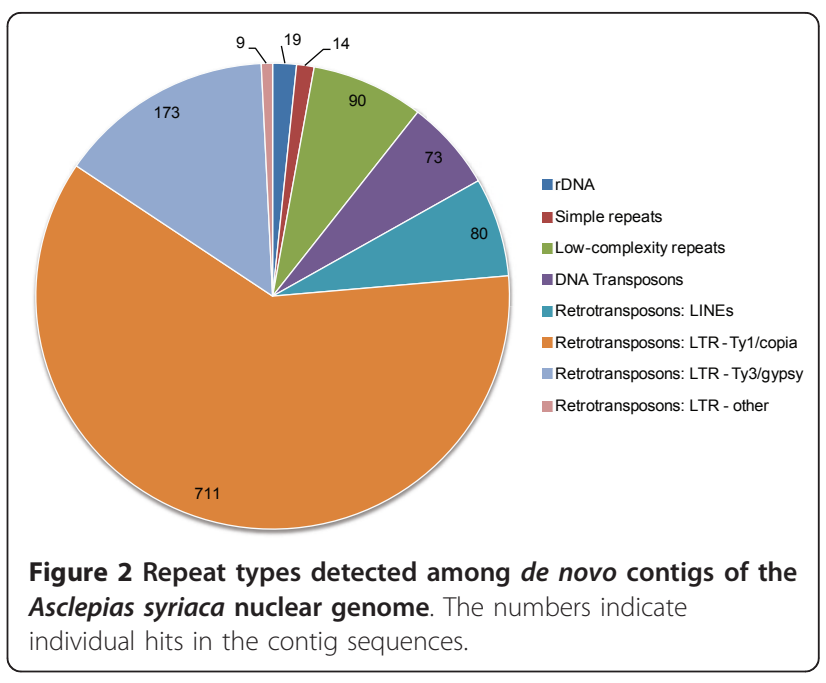

coverage to be assembled, so it is not surprising that the majority of the repeats were Ty1/copia-like and Ty3/ gypsy-like retrotransposons. In plant species where retrotransposons have been characterized, it appears to be lineage specific whether Ty1/copia-like or Ty3/gypsy-like retroelements are more abundant in the genome, likely because retrotransposons can proliferate or be lost from genomes over relatively short evolutionary time scales $[85,87]$. Among other asterids, Ty3/gypsy-like retrotransposons are more prevalent in the sunflower (Helianthus annuus), tomato, and potato genomes, while in carrot (Daucus carota), as in A. syriaca, Ty1/copia-like retrotransposons are more numerous [88-90].

The numbers of retroelements reported here are certain to be different than the actual numbers of retroelements in the sampled $A$. syriaca genome because the de novo contigs were relatively short compared to the length of retrotransposons, possibly resulting in different regions of the same retroelement being counted multiple times. Additionally, six contigs had more than one good repeat type match, slightly inflating the numbers discovered. Only approximately $2 \%$ of the putatively nuclear reads had hits to the repeat-containing contigs. The estimate of raw reads with hits to the plant repeat database did not give a better estimate of the total repeat content of the A. syriaca genome than the hits to assembled repeats with only $0.24 \%$ of reads with one or more hits. These results indicate that at $0.4 \times$ genome coverage, only an initial characterization of the most common repeats present in a plant genome can be made, while an estimation of the overall repeat content of the genome requires deeper coverage.

Microsatellite repeats were also detected among the 2,624 de novo contigs of at least $150 \mathrm{bp}$, with a total of 395 microsatellite sequences found in 333 of these contigs (Table 6). Top ten BLAST hits with an E-value of 
Table 6 Microsatellite loci detected in the $0.5 \times$ coverage genome of Asclepias syriaca

\begin{tabular}{cccc}
\hline Repeat Type & Number Found & Number of Loci with Primers & Number Tested/Successful for PCR Amplification \\
\hline di- & 271 & 206 & $17 / 17$ \\
tri- & 51 & 30 & $7 / 7$ \\
tet- & 57 & 43 & $1 / 1$ \\
pent- & 12 & 9 & $0 / N A$ \\
hex- & 4 & 2 & $0 / N A$ \\
\hline Total & $\mathbf{3 9 5}$ & $\mathbf{2 9 0}$ & $\mathbf{2 5 / 2 5}$ \\
\hline
\end{tabular}

Amplification success of the 25 markers with the highest numbers of repeat units is indicated (see Table 2).

$1 \mathrm{e}^{-5}$ or lower were recovered for 53 and three contigs for mitochondrial and chloroplast sequences respectively. The higher number of possible mitochondrial reads remaining in the read pool following removal of reads putatively belonging to the organellar genomes reflects our more limited knowledge of the mitochondrial genome in plants in general and incomplete assembly of the A. syriaca mitochondrial genome. An alternative explanation for the presence of these small segments of DNA of mitochondrial or chloroplast origin in putatively nuclear contigs is that they correspond to nuclear nomads [72].

To be conservative in selecting 25 candidates for testing, loci from the 56 contigs with putatively mitochondrial or chloroplast sequence were removed from the pool of candidates, leaving 209 primer sets for putatively nuclear microsatellite loci. See additional file 2: Primer sets designed using BatchPrimer3 for 184 nuclear microsatellite loci in Asclepias syriaca not tested for amplification success. Of the 25 primer sets tested, all successfully amplified in A. syriaca (Table 6). It is important to note that with the low overall genome coverage, at least some of these microsatellite-containing contigs are likely to have originated from multiple, nearly-identical paralogous loci. As is the case with all microsatellites, the ultimate proof in the utility of these markers for identifying intraspecific variation (e.g., linkage mapping or population genetics) will come from obeying a Mendelian locus/allele model that meets expected transmission ratios (in the case of known pedigrees) or Hardy-Weinberg expectations (in the case of wild populations). If the markers show evidence of fixed heterozygosity or dominant-only variation, this would constitute evidence that our microsatellite 'contigs' are the product of microreads representing two or more paralogs. These loci are currently being examined in greater detail to determine their locus specificity.

The source of the rDNA repeats identified by RepeatMasker in the de novo contigs (Figure 2) that remained after the removal of $A$. syriaca $\mathrm{rDNA}$ microreads was also explored. BLAST searches (blastn) of the NCBI nucleotide collection revealed that their source was likely plant fungal pathogens or endophytes. For example, the top BLAST hits for two of theses contigs were to an Alternaria alternata gene for large subunit (LSU) rRNA $\left(1 \mathrm{e}^{-105}\right)$ [GenBank:AB566324.1] and a Davidiella tassiana partial LSU rRNA gene sequence $\left(1 \mathrm{e}^{-92}\right)$ [GenBank: FN868880.1]. Approximately $0.02 \%$ of the total reads could be categorized as fungal rDNA when the sequences for the top GenBank hits were used as a BLAT database. Detection of fungal contaminants of a plant genomic DNA preparation is unsurprising due to the ubiquitous association of these two types of organisms.

\section{Ribosomal DNA}

A 385 bp contig containing a 120 bp 5S rDNA sequence [GenBank:JF312047] and an rDNA cistron of 7,541 bp [GenBank:JF312046] were assembled for $A$. syriaca (Figure 3$)$. Repeat structure in the external transcribed

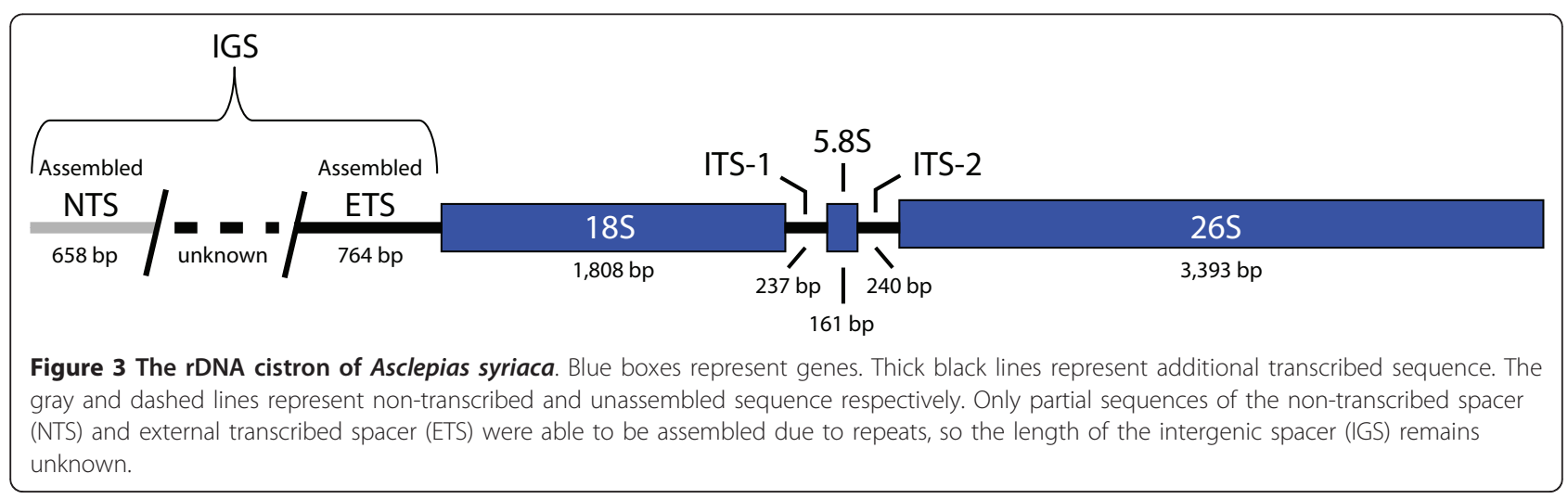


spacer (ETS) and intergenic spacer (IGS) regions of the rDNA cistron prevented further extension of that contig and produced an error in the assembly where reads corresponding to similar repeats were incorrectly piled up, highlighting one of the primary difficulties of genome assembly using short reads [32,91]. Consequently, the first $280 \mathrm{bp}$ of the contig sequence were removed prior to downstream analyses. The median read depth for the final assemblies were $406 \times$ for $5 \mathrm{~S}$ and $738 \times$ for the $18 \mathrm{~S}$ $5.8 \mathrm{~S}-26 \mathrm{~S}$ cistron. Using the median read depth as an estimate of the number of rDNA copies sequenced and the nuclear genome coverage of approximately $0.4 \times$, rough approximations of the number of $5 \mathrm{~S}$ rDNA repeats and of rDNA cistron copies are 1,015 and 1,845 respectively. These estimates are in line with values observed for other plants [92,93].

Fully characterized rDNA cistrons in plants have at least one TATA box upstream of $18 \mathrm{~S}$, which demarcates the boundary between the ETS region and the repeatrich non-transcribed spacer $[94,95]$. However, repeat units may also appear between the transcription initiation site (TIS) and 18S [96]. The conserved plant TIS sequence was not observed in the $A$. syriaca sequence upstream of $18 \mathrm{~S}$, so the entire ETS region was not assembled due to internal repeated sequence. However, another ETS motif conserved in plants and always located approximately 420 - 450 bp upstream of the start of 18S [TGAGTGGTGG; [97]] was located $423 \mathrm{bp}$ upstream of $18 \mathrm{~S}$ in the sequenced portion of ETS. Due to the length variation in the ETS regions in other asterids (Solanum 1000 bp; Nicotiana $>3000$ bp in N. tabacum; Olea $\sim 2000 \mathrm{bp}$ ), it is difficult to estimate the completeness of our assembly for this region and the cistron as a whole, which is between 9 and $12 \mathrm{~kb}$ in Nicotiana [96,98,99].

For intragenomic rDNA polymorphism characterization, quality filtering by average quality $(\mathrm{q}=20)$ removed zero reads from the $5 \mathrm{~S}$ pool, and 148 reads from the $18 \mathrm{~S}-5.8 \mathrm{~S}-26 \mathrm{~S}$ cistron pool $(0.1 \%)$. Masking the remaining nucleotides with qualities below 20 affected $1.7 \%$ and $6.2 \%$ of nucleotides in the two pools, respectively. A high percentage of positions in the $5 \mathrm{~S}$ locus were polymorphic (Figure 4). Most of these positions had less than $8 \%$ of reads differing from the consensus,

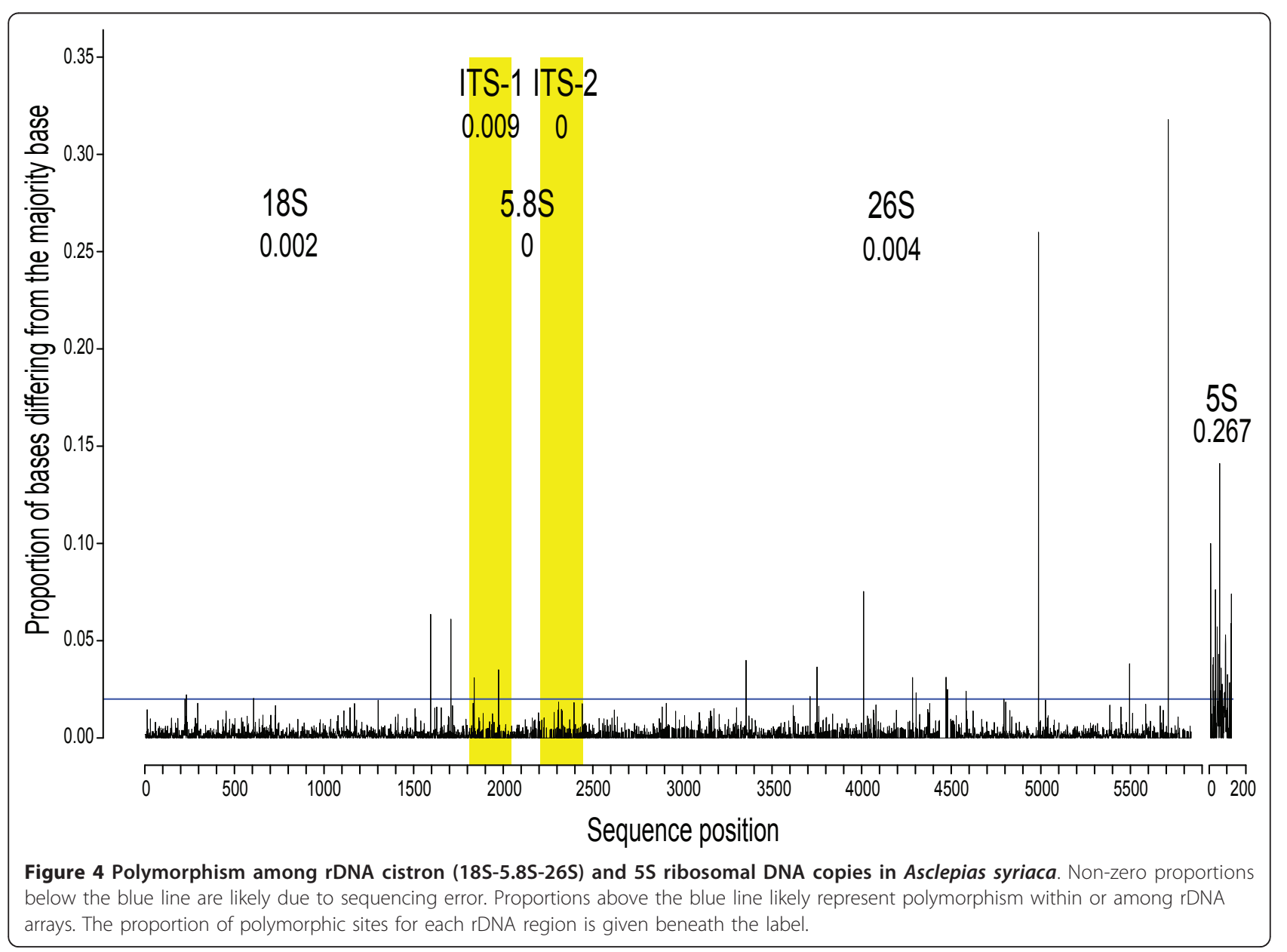


though some ranged as high as $14 \%$. Conversely, only 19 positions in the 18S-5.8S-26S cistron were polymorphic (Figure 4). Polymorphisms were found in both coding and spacer regions, although none were found in either the $5.8 \mathrm{~S}$ or ITS2 regions. While spacer regions contained a slightly higher proportion of polymorphic positions than coding regions, positions with the greatest proportion of differing reads were found within coding regions, in particular in the $26 \mathrm{~S}$ region.

Levels of intra-genomic polymorphism among copies of the rDNA loci generally agree with previously measured and theoretically expected levels. The high proportion of polymorphic positions in $5 \mathrm{~S}$ is concordant with previous observations for this locus; however, the levels measured here are somewhat higher than those reported in other plant and animal species [e.g., [100-102]]. Intragenomic polymorphism among copies of the rDNA cistron in this individual is comparable to rates found in previous studies characterizing polymorphism within Drosophila [103] and fungi [104]. While the 19 sites found in this study are within the previously observed range of variation, comparisons are difficult due to the difference in copy number between these taxa (45-180 in the fungi, 200-250 in Drosophila, approximately 1800 in this study), differences in the definition of polymorphic loci, and differences in sequencing technologies (wholegenome shotgun sequencing vs. Illumina).

The distribution of polymorphic positions matches theoretical expectations, with a higher proportion of positions found within spacer rather than coding regions. The very high proportion of differing reads found at two positions in the $26 \mathrm{~S}$ region may be caused by their location within expansion segments of this region, expansion segments 9 and 8, respectively [105]. The lower levels of polymorphism observed for the 18S$5.8 \mathrm{~S}-26 \mathrm{~S}$ cistron relative to the $5 \mathrm{~S}$ rDNA loci are concordant with the molecular evolutionary processes associated with each rDNA type. While strong concerted evolution and strong purifying selection act to homogenize the 18S-5.8S-26S cistron copies within and among repeat arrays, weak concerted evolution and birth-anddeath processes for $5 \mathrm{~S}$ rDNA allow accumulation of polymorphism $[100,106]$.

\section{Protein Coding Nuclear Genes}

From 19,899 Catharanthus ESTs, 9,266 unigenes were created. Of these, 72 corresponded to chloroplast, mitochondrial or rDNA genes and were removed from the data set. Among the remaining repeat masked unigenes, 8,077 had between one and 138 unique microread hits. The median number of hits per kilobase of sequence was 7.17 (95\% CI 7.03 - 7.29). The median coverage of the unigenes was $0.29 \times(95 \%$ CI $0.28 \times-0.29 \times$; Figure $5 \mathrm{a})$. The 618 genes with coverage levels above $0.984 \times$ were outliers. Many of these genes likely correspond to

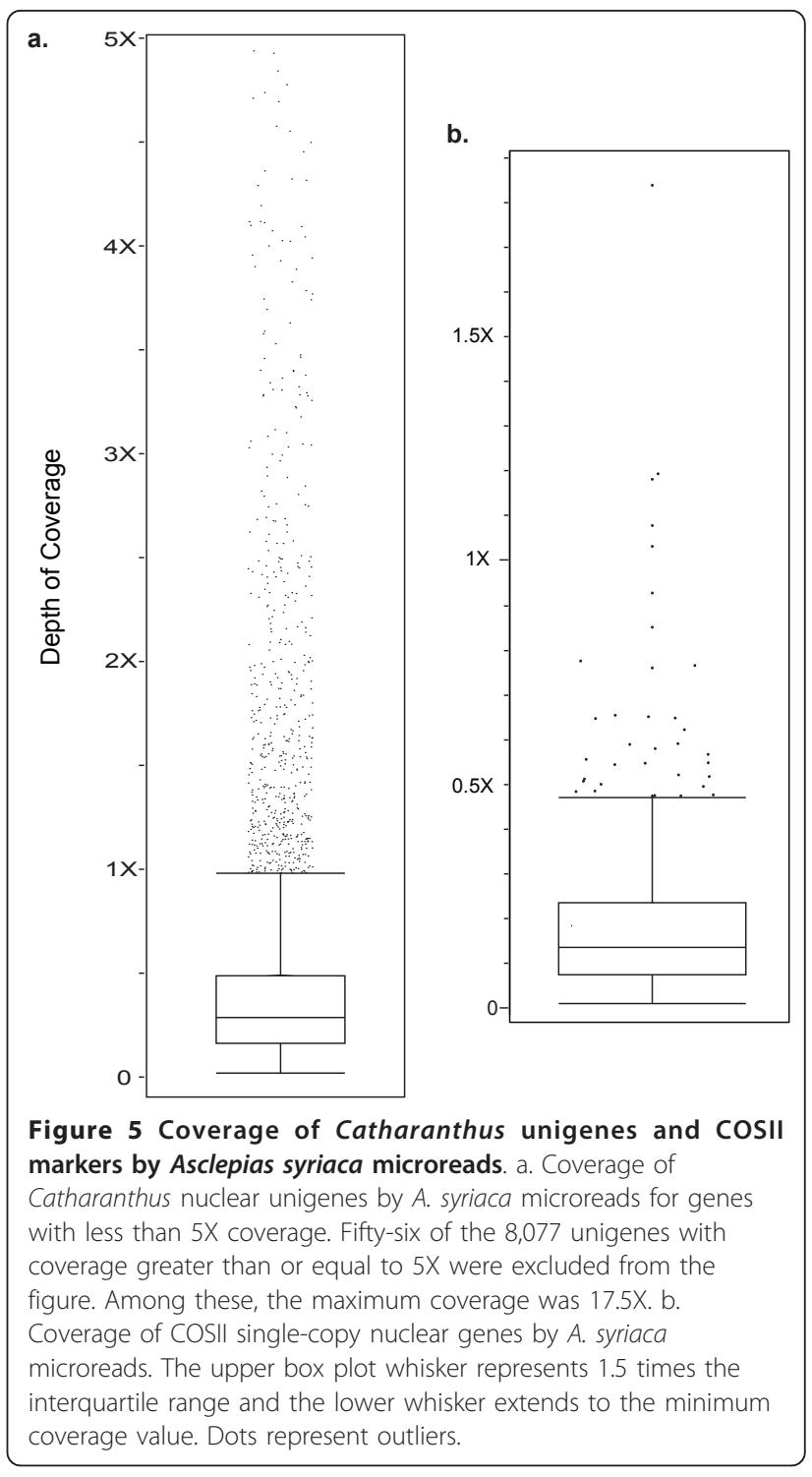

members of multigene families or other duplicated genes, increasing the chances of non-orthologous BLAT hits in conserved domains. For example, a unigene with $6.79 \times$ coverage had multiple BLAST hits $(\mathrm{E}=0)$ to eudicot $\mathrm{H}^{+}$-ATPases ( $\mathrm{P}_{3 \mathrm{~A}}$-type). Within the P-type ATPase gene super family, the $\mathrm{P}_{3 \mathrm{~A}}$-type subfamily has 10 and 11 members in rice and Arabidopsis respectively [107]. Based on the overall genome coverage and the unigene coverage, the $A$. syriaca genome likely contains a similar number of $\mathrm{P}_{3 \mathrm{~A}}$-type ATPases.

Another explanation for the high coverage of outlier loci was indicated by the masked unigene sequences themselves. Many of the unigenes with the highest coverage still contained sequences that could produce multiple non-homologous hits (e.g., internal polynucleotide stretches), which in turn produced stacks of reads. Read 
overlap due to this phenomenon inflated the coverage values, so many of the very high coverage values are likely artifacts of the unigene creation and masking process. The presence of PCR duplicate reads in the data set could also have caused read overlap and overestimation of coverage. Most of these reads should have been excluded by counting only unique BLAT hits, but any sequencing errors in the duplicates or length differences due to differing sequence quality, especially at the ends of the reads, could produce nearly identical hits.

Of the 1,086 COSII alignments 721 had between one and 23 unique $A$. syriaca microread hits with a median gene coverage of $0.14 \times(95 \%$ CI $0.13 \times-0.15 \times$; Figure $5 \mathrm{~b})$ and median hits per kilobase of sequence of 3.40 (95\% CI 3.13 - 3.64). The 35 genes with greater than $0.473 \times$ coverage were outliers. There is a possibility that some of these genes are single copy in other asterids, but duplicated in Asclepias, leading to the higher coverage levels. More likely, as in the unigene analysis, sequence features, including microsatellites that were not masked in the COSII alignments, and PCR duplicates led to increased estimates of coverage. Of the five genes with greater than $1 \times$ coverage, the only BLAT hits for two were to microsatellites and a third had multiple microsatellite and a single other hit.

The median coverage values for the unigene and COSII data sets would be expected to be close to the estimate of $0.4 \times$ coverage for the nuclear genome. However, the observed values deviated from the expected coverage. One explanation for this is that the overall coverage of the A. syriaca genome was slightly overestimated due to the presence of PCR duplicates in the read pool. It is also possible that the genome size of the sequenced individual was larger than the genome sizes estimated from the experimental population, again causing an overestimate of genome coverage. Divergence from the reference sequences would also cause an underestimate of coverage for A. syriaca orthologs. The lower amount of divergence from Catharanthus, a member of the same family, is reflected in the higher estimated coverage for the unigenes than the COSII alignments, which contained sequences of more distantly related asterids. The coverage value for the unigenes may also be higher due to the reasons discussed above. Another feature of both reference data sets that would cause an underestimate of coverage was the absence of introns. The microread sequences from genomic DNA would also represent these features of the genes, but would not produce BLAT hits for these genes. Reads spanning intron-exon boundaries would also have been excluded in many cases due to the mismatch of the intron sequence with the following exon in the unigenes and COSII alignments. Even with these issues confounding coverage estimates, these two explorations of the nuclear gene content of the A. syriaca genome demonstrate that even at $0.4 \times$ genome coverage, much of the protein coding portion of the nuclear genome can be detected.

Even with low overall coverage of the 721 COSII genes with $A$. syriaca microread hits, the alignments were still useful for marker development for Asclepias and Apocynaceae molecular phylogenetics. The 28 genes that had $0.5 \times$ or greater coverage were evaluated for their suitability for this purpose. Microreads flanked putative intron locations in 15 genes of this set, allowing primer design for amplification of one or more introns per gene (Table 3).

Primer testing within Asclepias and across Apocynaceae was largely successful with single products amplifying in most tribes of Apocynaceae (Table 7). Amplification of large introns and evidence of length variation among groups are promising for utility of these markers at different scales dependent upon overall sequence divergence. These results highlight how even a small amount of genomic data can facilitate and expedite the arduous process of nuclear marker development across a range of taxonomic scales from within a genus to across a family for groups of species not closely related to a species with extensive genomic resources.

\section{Conclusions}

The often-employed strategy of transcriptome sequencing for exploration of the genomes of non-model organisms can produce a wealth of information for comparative and functional genomics studies due to high coverage of the gene space and provide the necessary information for marker development, including single nucleotide polymorphisms [3]. As an alternative, low coverage whole genome sequencing provides a more complete survey of the entire genome by providing substantial information about the organellar genomes and the repeat content of the nuclear genome, while still providing the necessary resources for marker development. In plants with no prior genomic information, acquisition of even a small amount of genomic data allows ready characterization of the high copy fraction of the genome and exploration of the low copy fraction of the genome. A complete, or nearly complete, chloroplast genome and rDNA cistron are readily obtained. A partial to nearly complete mitochondrial genome can be obtained, depending on the complexity of the particular mitochondrial genome. Initial characterizations of the repeat and protein coding portions of the nuclear genome can be made in terms of content and then used in the development of molecular tools, including low-copy nuclear genes and microsatellites, for the further study of the target organism and its relatives. 
Table 7 Success of PCR amplification and intron length variation for COSII nuclear loci in Asclepias and across Apocynaceae

\begin{tabular}{|c|c|c|c|c|c|c|c|c|c|c|c|c|c|c|}
\hline \multirow[b]{2}{*}{$\begin{array}{l}\text { Arabidopsis } \\
\text { Ortholog }\end{array}$} & \multirow[b]{2}{*}{ tair Annotation } & \multirow[b]{2}{*}{ Primer Set } & \multirow[b]{2}{*}{$\begin{array}{l}\text { Introns } \\
\text { spanned* }\end{array}$} & \multirow[b]{2}{*}{$\begin{array}{l}\text { Coding } \\
\text { Sequence } \\
\text { (bp) }\end{array}$} & \multicolumn{5}{|c|}{$\begin{array}{l}\text { Number of Individuals PCR } \\
\text { successful/PCR attempted }\end{array}$} & \multicolumn{5}{|c|}{ Approximate Product Size (bp)\# } \\
\hline & & & & & $\mathrm{As}^{1}$ & $M^{2}$ & $B^{3}$ & $A p^{4}$ & $\mathrm{Al}^{5}$ & As & $M$ & B & Ap & Al \\
\hline \multirow[t]{3}{*}{ At1g24360 } & $\begin{array}{l}\text { 3-oxoacyl-(acyl-carrier protein) } \\
\text { reductase, chloroplast }\end{array}$ & At1g24360a & $360,441,487$ & 234 & $15 / 20$ & $1 / 2$ & $1 / 2$ & $2 / 2$ & $1 / 1$ & $1400-1500$ & 1500 & 1000 & 300,1000 & 1000 \\
\hline & & At1g24360b & 582,615 & 117 & $14 / 16$ & $2 / 2$ & $2 / 2$ & $2 / 2$ & $1 / 1$ & $500-700$ & 500 & 500 & 500 & $\begin{array}{l}700 \\
1500\end{array}$ \\
\hline & & At1g24360c & 750 & 117 & $16 / 16$ & $2 / 2$ & $2 / 2$ & $2 / 2$ & $1 / 1$ & 200 & 200 & 200,1500 & 200 & 200,700 \\
\hline At1g44760 & $\begin{array}{l}\text { universal stress protein (USP) family } \\
\text { protein }\end{array}$ & At1g44760a & 369,491 & 334 & $1 / 16$ & $1 / 2$ & $1 / 2$ & $2 / 2$ & $1 / 1$ & 1300 & 1200 & 1200 & 1100 & 700 \\
\hline At1g55880 & $\begin{array}{l}\text { pyridoxal-5'-phosphate-dependent } \\
\text { enzyme }\end{array}$ & At1g55880a & 1043 & 394 & $13 / 16$ & $2 / 2$ & $2 / 2$ & $2 / 2$ & $1 / 1$ & 1200 & 900 & $700-800$ & 700 & 300,700 \\
\hline At1g77370 & glutaredoxin, putative & At1g77370a & 164,238 & 172 & $16 / 20$ & $2 / 2$ & $2 / 2$ & $0 / 2$ & $0 / 1$ & $1400-1500$ & 1100 & $1100-1200$ & $\mathrm{n} / \mathrm{a}$ & $\mathrm{n} / \mathrm{a}$ \\
\hline At2g03120 & $\begin{array}{l}\text { homologous to Signal Peptide } \\
\text { Peptidases (SPP) }\end{array}$ & At2g03120a & 156 & 210 & $19 / 19$ & $2 / 2$ & $2 / 2$ & $2 / 2$ & $1 / 1$ & $300-400$ & 300 & 300 & 300 & 300 \\
\hline At2g06530 & VPS2.1 (vesicle-mediated transport) & At2g06530a & 276 & 145 & $20 / 20$ & $2 / 2$ & $2 / 2$ & $2 / 2$ & $1 / 1$ & $400-650$ & 400 & $\begin{array}{c}700- \\
(600,1000)\end{array}$ & $\begin{array}{c}600- \\
(800,1000)\end{array}$ & $\begin{array}{l}400 \\
1200\end{array}$ \\
\hline \multirow[t]{2}{*}{ At2g21960 } & unknown protein & At2g21960a & $503,592,722$ & 366 & $7 / 16$ & $2 / 2$ & $2 / 2$ & $2 / 2$ & $1 / 1$ & 1500 & 1500 & 1700 & $\begin{array}{l}1600- \\
1700\end{array}$ & 1100 \\
\hline & & At2g21960b & 813 & 84 & $12 / 16$ & $2 / 2$ & $2 / 2$ & $2 / 2$ & $1 / 1$ & $200-300$ & 200 & 150 & 150 & 150 \\
\hline \multirow[t]{3}{*}{ At2g30200 } & $\begin{array}{l}\text { [acyl-carrier-protein] S- } \\
\text { malonyltransferase }\end{array}$ & At2g30200a & 377,444 & 185 & $12 / 16$ & $2 / 2$ & $2 / 2$ & $2 / 2$ & $1 / 1$ & 1400 & 1000 & 1200 & 900 & $\begin{array}{l}1200 \\
>>1500\end{array}$ \\
\hline & & At2g30200b & $587,636,774$ & 392 & $13 / 20$ & $2 / 2$ & $2 / 2$ & $2 / 2$ & $1 / 1$ & 700-1500 & 1200 & 1200 & 1200 & 1200 \\
\hline & & At2g30200c & 855 & 160 & $16 / 16$ & $0 / 2$ & $2 / 2$ & $2 / 2$ & $1 / 1$ & 300 & $\mathrm{n} / \mathrm{a}$ & 300 & 300 & 300 \\
\hline \multirow[t]{3}{*}{ At2g34620 } & $\begin{array}{l}\text { mitochondrial transcription } \\
\text { termination factor-related }\end{array}$ & At2g34620a & 596 & 254 & $19 / 19$ & $2 / 2$ & $2 / 2$ & $2 / 2$ & $2 / 2$ & 300 & 300 & 300 & $\begin{array}{l}300- \\
1000\end{array}$ & 300 \\
\hline & & At2g34620F/ & 596 & 531 & $15 / 16$ & $2 / 2$ & $2 / 2$ & $2 / 2$ & $0 / 2$ & 600 & 600 & 600 & 600 & $\mathrm{n} / \mathrm{a}$ \\
\hline & & At2g34620aR & & & & & & & & & & & & \\
\hline \multirow[t]{2}{*}{ At2g47390 } & serine-type endopeptidase & At2g47390a & 1718 & 401 & $20 / 20$ & $2 / 2$ & $2 / 2$ & $2 / 2$ & $0 / 2$ & $500-600$ & 500 & 500 & 500 & $\mathrm{n} / \mathrm{a}$ \\
\hline & & At2g47390b & $\begin{array}{l}2177,2243, \\
2304,2454, \\
2592\end{array}$ & 506 & $1 / 16$ & $0 / 2$ & $0 / 2$ & $0 / 2$ & $0 / 2$ & 1500 & $\mathrm{n} / \mathrm{a}$ & $\mathrm{n} / \mathrm{a}$ & $\mathrm{n} / \mathrm{a}$ & $\mathrm{n} / \mathrm{a}$ \\
\hline \multirow[t]{3}{*}{ At4g13430 } & methylthioalkylmalate isomerase & At4g13430a & $510,579,696$ & 275 & $14 / 16$ & $2 / 2$ & $2 / 2$ & $2 / 2$ & $1 / 1$ & $700-1000$ & $\begin{array}{l}900 \\
1200\end{array}$ & 1200 & 1200 & 1200 \\
\hline & & At4g13430b & $\begin{array}{l}771,849,945 \\
995,1050\end{array}$ & 391 & $16 / 20$ & $0 / 2$ & $2 / 2$ & $0 / 2$ & $1 / 1$ & $1300-1500$ & $n / a$ & $>1500$ & $\mathrm{n} / \mathrm{a}$ & 1500 \\
\hline & & At4g13430c & 1182,1230 & 278 & $15 / 16$ & $2 / 2$ & $2 / 2$ & $2 / 2$ & $1 / 1$ & $\begin{array}{l}(500,800)- \\
1000\end{array}$ & 800 & $\begin{array}{l}900- \\
>1500\end{array}$ & 800 & $\begin{array}{l}500,900 \\
1200\end{array}$ \\
\hline
\end{tabular}


Table 7 Success of PCR amplification and intron length variation for COSII nuclear loci in Asclepias and across Apocynaceae (Continued)

\begin{tabular}{|c|c|c|c|c|c|c|c|c|c|c|c|c|c|c|}
\hline At4g26750 & $\begin{array}{l}\text { hydroxyproline-rich glycoprotein } \\
\text { family protein }\end{array}$ & At4g26750a & 305,381 & 241 & $1 / 16$ & $2 / 2$ & $2 / 2$ & $2 / 2$ & $0 / 1$ & 1500 & $>1500$ & 1500 & 1000 & $\mathrm{n} / \mathrm{a}$ \\
\hline \multirow[t]{2}{*}{ At5g13420 } & putative transaldolase & At5g13420a & 348,398 & 295 & $12 / 16$ & $2 / 2$ & $2 / 2$ & $2 / 2$ & $0 / 1$ & 1300 & 1100 & 1000 & 700 & $\mathrm{n} / \mathrm{a}$ \\
\hline & & At5g13420b & 699,888 & 338 & $20 / 20$ & $2 / 2$ & $2 / 2$ & $2 / 2$ & $1 / 1$ & $800-900$ & 1200 & 1200 & 500,1200 & 1500 \\
\hline \multirow[t]{2}{*}{ At5g23540 } & 265 proteasome regulatory subunit & At5g23540a & $308,390,567$ & 436 & $0 / 16$ & $2 / 2$ & $2 / 2$ & $1 / 2$ & $0 / 1$ & n/a & $>>1500$ & $\begin{array}{l}300,700 \\
>>1500\end{array}$ & 500,700 & $\mathrm{n} / \mathrm{a}$ \\
\hline & & $\begin{array}{l}\text { At5g23540aF } \\
\text { At5g23540bR }\end{array}$ & 308,390 & 231 & $7 / 16$ & $2 / 2$ & $2 / 2$ & $1 / 2$ & $1 / 1$ & 1400 & $>>1500$ & $\begin{array}{l}700-900 \\
>>1500\end{array}$ & 700 & 1200 \\
\hline \multirow[t]{3}{*}{ At5g49970 } & $\begin{array}{l}\text { bifunctional pyridoxine } \\
\text { (pyridoxamine) 5'-phosphate } \\
\text { oxidase (PPOX) }\end{array}$ & At5g49970a & 507 & 193 & $20 / 20$ & $2 / 2$ & $2 / 2$ & $2 / 2$ & $2 / 2$ & 300 & 300 & 300 & 300 & 300 \\
\hline & & At5g49970b & 613,780 & 226 & $16 / 16$ & $2 / 2$ & $2 / 2$ & $2 / 2$ & $1 / 2$ & 500-900 & 800 & 800 & 700 & 1000 \\
\hline & & At5g49970aFAt5g49970bR & $507,613,780$ & 398 & $15 / 16$ & $n / a$ & $\mathrm{n} / \mathrm{a}$ & $\mathrm{n} / \mathrm{a}$ & $\mathrm{n} / \mathrm{a}$ & 1000-1500 & $\mathrm{n} / \mathrm{a}$ & $\mathrm{n} / \mathrm{a}$ & $\mathrm{n} / \mathrm{a}$ & $\mathrm{n} / \mathrm{a}$ \\
\hline
\end{tabular}

*Intron numbers represent the starting position of the intron in Arabidopsis.

'Asclepiadinae Samples include 4 genera: Asclepias (17), Gomphocarpus, Calotropis, and Pergularia.

${ }^{2}$ Marsdenieae

${ }^{3}$ Baisseeae

${ }^{4}$ Apocyneae

${ }^{5}$ Alyxieae

\#Two numbers separated by a comma indicate multiple PCR products. A range of numbers indicates size variation among individuals. 
The data obtained through this study are the first step in the development of a community resource for further study of plant-herbivore and plant-pollinator interactions, floral developmental genetics, chemical evolution, population genetics, and comparative genomics using milkweeds as ecological and evolutionary models. The information obtained from this study is already serving as a resource for development of markers to aid in the study of phylogenetics, population genetics, phylogeography and other areas for Asclepias and Apocynaceae. These results highlight the promise of genomic resources for developing tools in any organism $[2,108]$ and show that even labs with limited resources can feasibly obtain $0.5 \times$ or greater coverage of the genome of their species of interest and a wealth of information. By incorporating 80-120 bp read lengths, paired-end sequences, and ever-improving sequencing technology, this level of coverage and assembly success from a single lane of Illumina sequencing is attainable for all but the very largest of plant genomes.

\section{Additional material}

Additional file 1: Polymorphism detected among Illumina reads for the PhiX sequencing control. A graph showing the proportion of bases differing from the majority base in the Illumina PhiX sequencing control.

Additional file 2: Primer sets designed using BatchPrimer3 for 184 nuclear microsatellite loci in Asclepias syriaca not tested for amplification success. A table of nuclear microsatellite locus primer sets, including predicted product size and repeat motif information.

Additional file 3: List of GI numbers for 19,899 Catharanthus roseus ESTs downloaded from GenBank and assembled into unigenes. List of GenBank numbers for Catharanthus roseus ESTs used in this study.

Additional file 4: Asclepias syriaca mitochondrial genome contigs. FASTA file containing sequences of the assembled Asclepias syriaca mitochondrial genome contigs.

Additional file 5: Asclepias syriaca nuclear genome de novo assembly contigs. FASTA file containing sequences of the nuclear genome contigs from the Velvet de novo Asclepias syriaca genome assembly.

\section{Acknowledgements}

The authors thank Michael Moore and Douglas Soltis for providing the unpublished Nerium oleander chloroplast genome sequence, Winthrop Phippen for supplying the tissue used for genome size estimation and sequence comparisons, Chris Poklemba for collecting the flow cytometry data, Brian Knaus for access to data processing scripts, Christopher Sullivan for informatics infrastructure management and assistance, Mark Dasenko for Illumina sequencing support, LaRinda Holland, Wyatt Sharber, Angela Rein, and Kevin Kilhoffer for laboratory assistance, and Aakrosh Ratan for YASRA access and support. This research was funded by a grant from the U.S. National Science Foundation Systematic Biology Program (DEB 0919583) to $\mathrm{RCC}, \mathrm{MF}$, and AL.

\section{Author details}

'Department of Botany and Plant Pathology, Oregon State University, 2082 Cordley Hall, Corvallis, Oregon 97331, USA. ${ }^{2}$ Department of Botany,

Oklahoma State University, 104 Life Sciences East, Stillwater, Oklahoma 74078, USA. ${ }^{3}$ The Academy of Natural Sciences, 1900 Benjamin Franklin Parkway, Philadelphia, Pennsylvania 19103, USA. ${ }^{4}$ Pacific Northwest Research
Station, USDA Forest Service, 3200 SW Jefferson Way, Corvallis, Oregon 97331, USA.

\section{Authors' contributions}

SCKS participated in study design, conducted the majority of the analyses, designed the primers, collected the chloroplast Sanger sequence data and drafted the manuscript. MF conceived of the study and conducted microsatellite and low-copy nuclear gene primer testing in Asclepias. TL participated in the design of the low-copy nuclear gene analyses and conducted low-copy nuclear gene primer testing in Apocynaceae. ZF scripted and tested the data analysis pipeline. MP prepared the Illumina library and tested the data analysis pipeline. KW conducted the rDNA polymorphism analyses. RCC conceived of the study, performed some statistical analyses, and collected the genome size data. AL conceived of the study, coordinated the data collection and study design, conducted the COSII nuclear gene analysis and assisted in some of the other analyses. All authors read and approved the final manuscript.

Received: 26 February 2011 Accepted: 4 May 2011

Published: 4 May 2011

\section{References}

1. Rokas A, Abbot P: Harnessing genomics for evolutionary insights. Trends Ecol Evol 2009, 24(4):192-200.

2. Hudson ME: Sequencing breakthroughs for genomic ecology and evolutionary biology. Mol Ecol Resour 2008, 8(1):3-17.

3. Ekblom R, Galindo J: Applications of next generation sequencing in molecular ecology of non-model organisms. Heredity.

4. Rasmussen DA, Noor MAF: What can you do with $0.1 \times$ genome coverage? A case study based on a genome survey of the scuttle fly Megaselia scalaris (Phoridae). BMC Genomics 2009, 10:382.

5. Lee RM, Thimmapuram J, Thinglum KA, Gong G, Hernandez AG, Wright $C L$, Kim RW, Mikel MA, Tranel PJ: Sampling the Waterhemp (Amaranthus tuberculatus) genome using pyrosequencing technology. Weed Sci 2009, 57(5):463-469.

6. Swaminathan K, Alabady MS, Varala K, De Paoli E, Ho I, Rokhsar DS, Arumuganathan AK, Ming R, Green PJ, Meyers BC, et al: Genomic and small RNA sequencing of Miscanthus $\times$ giganteus shows the utility of sorghum as a reference genome sequence for Andropogoneae grasses. Genome Biol 2010, 11(2):R12.

7. Hribova E, Neumann P, Matsumoto T, Roux N, Macas J, Dolezel J: Repetitive part of the banana (Musa acuminata) genome investigated by lowdepth 454 sequencing. BMC Plant Biol 2010, 10:204.

8. Macas J, Neumann P, Navratilova A: Repetitive DNA in the pea (Pisum sativum L.) genome: comprehensive characterization using 454 sequencing and comparison to soybean and Medicago truncatula. BMC Genomics 2007, 8:427.

9. Webb KM, Rosenthal BM: Next-generation sequencing of the Trichinella murrelli mitochondrial genome allows comprehensive comparison of its divergence from the principal agent of human trichinellosis, Trichinella spiralis. Infect Genet Evol 2011, 11(1):116-123.

10. Willerslev E, Gilbert MT, Binladen J, Ho S, Campos P, Ratan A, Tomsho L, da Fonseca R, Sher A, Kuznetsova T, et al: Analysis of complete mitochondrial genomes from extinct and extant rhinoceroses reveals lack of phylogenetic resolution. BMC Evol Biol 2009, 9(1):95.

11. Dempewolf H, Kane NC, Ostevik KL, Geleta M, Barker MS, Lai Z, Stewart ML, Bekele E, Engels JMM, Cronk QCB, et al: Establishing genomic tools and resources for Guizotia abyssinica (L.f.) Cass.-the development of a library of expressed sequence tags, microsatellite loci, and the sequencing of its chloroplast genome. Mol Ecol Resour 2010, 10(6):1048-1058

12. Meyers $\mathrm{S}$, Liston $\mathrm{A}$ : Characterizing the genome of wild relatives of Limnanthes alba (Meadowfoam) using massively parallel sequencing. Acta Horticulturae 2010, 859:309-314.

13. Nock CJ, Waters DL, Edwards MA, Bowen SG, Rice N, Cordeiro GM, Henry RJ: Chloroplast genome sequences from total DNA for plant identification. Plant Biotechnol J 2011, 9:328-333.

14. Givnish TJ, Ames M, McNeal JR, McKain MR, Steele PR, dePamphilis CW, Graham SW, Pires JC, Stevenson DW, Zomlefer WB, et al: Assembling the tree of the monocotyledons: plastome sequence phylogeny and evolution of Poales 1. Ann Mo Bot Gard 2010, 97(4):584-616. 
15. Castoe TA, Poole AW, Gu WJ, de Koning APJ, Daza JM, Smith EN, Pollock DD: Rapid identification of thousands of copperhead snake (Agkistrodon contortrix) microsatellite loci from modest amounts of 454 shotgun genome sequence. Mol Ecol Resour 2010, 10(2):341-347.

16. Abdelkrim J, Robertson BC, Stanton JAL, Gemmell NJ: Fast, cost-effective development of species-specific microsatellite markers by genomic sequencing. Biotechniques 2009, 46(3):185-192.

17. Moore MJ, Soltis PS, Bell CD, Burleigh JG, Soltis DE: Phylogenetic analysis of 83 plastid genes further resolves the early diversification of eudicots. Proc Natl Acad Sci USA 2010, 107(10):4623-4628.

18. Bai $X$, Zhang W, Orantes L, Jun T-H, Mittapalli O, Mian MAR, Michel AP: Combining next-generation sequencing strategies for rapid molecular resource development from an invasive aphid species, Aphis glycines. PLOS ONE 2010, 5(6):e11370..

19. Rasmann S, Agrawal AA, Cook SC, Erwin AC: Cardenolides, induced responses, and interactions between above- and belowground herbivores of milkweed (Asclepias spp.). Ecology 2009, 90(9):2393-2404.

20. Broyles SB: Hybrid bridges to gene flow: A case study in milkweeds (Asclepias). Evolution 2002, 56(10):1943-1953.

21. Wyatt R, Broyles SB: Ecology and evolution of reproduction in milkweeds. Annu Rev Ecol Syst 1994, 25:423-441.

22. Fishbein $M$, Venable DL: Evolution of inflorescence design: Theory and data. Evolution 1996, 50(6):2165-2177.

23. Agrawal AA, Fishbein M: Phylogenetic escalation and decline of plant defense strategies. Proc Natl Acad Sci USA 2008, 105(29):10057-10060.

24. Agrawal AA, Fishbein M: Plant defense syndromes. Ecology 2006, 87(7): S132-S149

25. Malcolm SB: Cardenolide-mediated interactions between plants and herbivores. In Herbivores: their interactions with secondary plant metabolites Volume l: The chemical participants.. 2 edition. Edited by: Rosenthal GA, Berenbaum MR. San Diego: Academic Press; 1991:251-296.

26. Murata J, Bienzle D, Brandle JE, Sensen CW, De Luca V: Expressed sequence tags from Madagascar periwinkle (Catharanthus roseus). FEBS Lett 2006, 580(18):4501-4507.

27. Murata J, Roepke J, Gordon H, De Luca V: The leaf epidermome of Catharanthus roseus reveals its biochemical specialization. Plant Cell 2008, 20(3):524-542.

28. Shukla AK, Shasany AK, Gupta MM, Khanuja SPS: Transcriptome analysis in Catharanthus roseus leaves and roots for comparative terpenoid indole alkaloid profiles. J Exp Bot 2006, 57(14):3921-3932

29. Abzhanov A, Extavour CG, Groover A, Hodges SA, Hoekstra HE, Kramer EM, Monteiro A: Are we there yet? Tracking the development of new model systems. Trends in Genet 2008, 24(7):353-360.

30. Dolezel J, Bartos J, Voglmayr H, Greilhuber J: Nuclear DNA content and genome size of trout and human. Cytom Part A 2003, 51A(2):127-128.

31. Solexa, Inc: Protocol for Whole Genome Sequencing using Solexa Technology. BioTechniques Protocol Guide 2007 New York: BioTechniques; 2006, 291

32. Ratan A: Assembly algorithms for next-generation sequence data. PhD thesis The Pennsylvania State University, Computer Science and Engineering; 2009.

33. Delcher AL, Phillippy A, Carlton J, Salzberg SL: Fast algorithms for largescale genome alignment and comparison. Nucleic Acids Res 2002, 30(11):2478-2483.

34. Kurtz S, Phillippy A, Delcher AL, Smoot M, Shumway M, Antonescu C, Salzberg SL: Versatile and open software for comparing large genomes. Genome Biol 2004, 5(2):R12.

35. Ovcharenko I, Loots GG, Giardine BM, Hou MM, Ma J, Hardison RC, Stubbs L, Miller W: Mulan: Multiple-sequence local alignment and visualization for studying function and evolution. Genome Res 2005, 15(1):184-194.

36. Katoh K, Kuma K, Toh H, Miyata T: MAFFT version 5: improvement in accuracy of multiple sequence alignment. Nucleic Acids Res 2005, 33(2):511-518.

37. Wyman SK, Jansen RK, Boore JL: Automatic annotation of organellar genomes with DOGMA. Bioinformatics 2004, 20(17):3252-3255.

38. Milne I, Bayer M, Cardle L, Shaw P, Stephen G, Wright F, Marshall D: Tabletnext generation sequence assembly visualization. Bioinformatics 2010, 26(3):401-402.

39. Zerbino D, Birney E: Velvet: algorithms for de novo short read assembly using de Bruijn graphs. Genome Res 2008, 18:821-829.
40. Hall TA: BioEdit: a user-friendly biological sequence alignment editor and analysis program for Windows 95/98/NT. Nucl Acids Symp Ser 1999, 41:95-98.

41. Conant GC, Wolfe KH: GenomeVx: simple web-based creation of editable circular chromosome maps. Bioinformatics 2008, 24(6):861-862

42. Sugiyama $Y$, Watase $Y$, Nagase M, Makita N, Yagura S, Hirai A, Sugiura M The complete nucleotide sequence and multipartite organization of the tobacco mitochondrial genome: comparative analysis of mitochondrial genomes in higher plants. Mol Genet Genomics 2005, 272(6):603-615.

43. Alverson AJ, Wei XX, Rice DW, Stern DB, Barry K, Palmer JD: Insights into the evolution of mitochondrial genome size from complete sequences of Citrullus lanatus and Cucurbita pepo (Cucurbitaceae). Mol Biol Evol 2010, 27(6):1436-1448.

44. Kent WJ: BLAT - The BLAST-like alignment tool. Genome Res 2002, 12(4):656-664.

45. Knaus B: Short read toolbox.[http://brianknaus.com].

46. Gordon A: FASTX-Toolkit.[http://hannonlab.cshl.edu/fastx_toolkit/index. html].

47. Li H, Durbin R: Fast and accurate short read alignment with BurrowsWheeler transform. Bioinformatics 2009, 25(14):1754-1760.

48. Nguyen P, Ma J, Pei D, Obert C, Cheng C, Geiger T: Identification of errors introduced during high throughput sequencing of the $T$ cell receptor repertoire. BMC Genomics 2011, 12(1):106..

49. Gladman S, Seeman T: VelvetOptimiser version 2.1.7.[http://bioinformatics. net.au/software.velvetoptimiser.shtml]

50. Cantarel BL, Korf I, Robb SMC, Parra G, Ross E, Moore B, Holt C, Alvarado AS, Yandell M: MAKER: An easy-to-use annotation pipeline designed for emerging model organism genomes. Genome Res 2008, 18(1):188-196.

51. Smit A, Hubley R, Green P: RepeatMasker Open-3.0.[http://www. repeatmasker.org]

52. Jurka J, Kapitonov W, Pavlicek A, Klonowski P, Kohany O, Walichiewicz J: Repbase update, a database of eukaryotic repetitive elements. Cytogenet Genome Res 2005, 110(1-4):462-467

53. You FM, Huo NX, Gu YQ, Luo MC, Ma YQ, Hane D, Lazo GR, Dvorak J, Anderson OD: BatchPrimer3: A high throughput web application for PCR and sequencing primer design. BMC Bioinformatics 2008, 9:253.

54. He J, Dai XB, Zhao XC: PLAN: a web platform for automating highthroughput BLAST searches and for managing and mining results. BMC Bioinformatics 2007, 8:53

55. Altschul SF, Madden TL, Schaffer AA, Zhang JH, Zhang Z, Miller W Lipman DJ: Gapped BLAST and PSI-BLAST: a new generation of protein database search programs. Nucleic Acids Res 1997, 25(17):3389-3402.

56. Hood GM: PopTools version 3.2.3.[http://www.poptools.org].

57. Wu F, Mueller LA, Crouzillat D, Petiard V, Tanksley SD: Combining bioinformatics and phylogenetics to identify large sets of single-copy orthologous genes (COSII) for comparative, evolutionary and systematic studies: a test case in the euasterid plant clade. Genetics 2006, 174(3):1407-1420.

58. Livshultz T, Middleton DJ, Endress ME, Williams JK: Phylogeny of Apocynoideae and the APSA clade (Apocynaceae s.l.). Ann Mo Bot Gard 2007, 94(2):324-359.

59. Angiosperm DNA C-values Database (Release 6.0, Oct. 2005). [http:// www.kew.org/cvalues].

60. Meve U: Species numbers and progress in Asclepiad taxonomy. Kew Bull 2002, 57(2):459-464.

61. Ravi V, Khurana JP, Tyagi AK, Khurana P: An update on chloroplast genomes. Plant Syst Evol 2008, 271(1-2):101-122

62. Samson N, Bausher MG, Lee SB, Jansen RK, Daniell H: The complete nucleotide sequence of the coffee (Coffea arabica L.) chloroplast genome: organization and implications for biotechnology and phylogenetic relationships amongst angiosperms. Plant Biotechnol J 2007, 5(2):339-353.

63. Jansen RK, Wojciechowski MF, Sanniyasi E, Lee SB, Daniell H: Complete plastid genome sequence of the chickpea (Cicer arietinum) and the phylogenetic distribution of rps12 and clpP intron losses among legumes (Leguminosae). Mol Phylogenet Evol 2008, 48(3):1204-1217.

64. Lee HL, Jansen RK, Chumley TW, Kim KJ: Gene relocations within chloroplast genomes of Jasminum and Menodora (Oleaceae) are due to multiple, overlapping inversions. Mol Biol Evol 2007, 24(5):1161-1180.

65. Guisinger MM, Chumley TW, Kuehl JV, Boore JL, Jansen RK: Implications of the plastid genome sequence of Typha (Typhaceae, Poales) for 
understanding genome evolution in Poaceae. J Mol Evol 2010, 70(2):149-166.

66. Greiner S, Wang X, Rauwolf U, Silber MV, Mayer K, Meurer J, Haberer G, Herrmann RG: The complete nucleotide sequences of the five genetically distinct plastid genomes of Oenothera, subsection Oenothera: I. Sequence evaluation and plastome evolution. Nucleic Acids Res 2008, 36(7):2366-2378

67. Guisinger MM, Kuehl JV, Boore $J \mathrm{~L}$, Jansen RK: Extreme reconfiguration of plastid genomes in the angiosperm family Geraniaceae: Rearrangements, repeats, and codon usage. Mol Biol Evol 2011, 28(1):583-600.

68. Jansen RK, Cai Z, Raubeson LA, Daniell H, dePamphilis CW, Leebens-Mack J, Müller KF, Guisinger-Bellian M, Haberle RC, Hansen AK, et al: Analysis of 81 genes from 64 plastid genomes resolves relationships in angiosperms and identifies genome-scale evolutionary patterns. Proc Natl Acad Sci USA 2007, 104(49):19369-19374

69. Haberle RC, Fourcade HM, Boore $J$, Jansen RK: Extensive rearrangements in the chloroplast genome of Trachelium caeruleum are associated with repeats and tRNA genes. J Mol Evol 2008, 66(4):350-361.

70. Jo Y, Park J, Kim J, Song W, Hur C-G, Lee Y-H, Kang B-C: Complete sequencing and comparative analyses of the pepper (Capsicum annuum L.) plastome revealed high frequency of tandem repeats and large insertion/deletions on pepper plastome. Plant Cell Rep 2010, 1-13.

71. Cai ZQ, Guisinger M, Kim HG, Ruck E, Blazier JC, McMurtry V, Kuehl JV, Boore J, Jansen RK: Extensive reorganization of the plastid genome of Trifolium subterraneum (Fabaceae) is associated with numerous repeated sequences and novel DNA insertions. J Mol Evol 2008, 67(6):696-704.

72. Arthofer W, Schuler S, Steiner FM, Schlick-Steiner BC: Chloroplast DNAbased studies in molecular ecology may be compromised by nuclearencoded plastid sequence. Mol Ecol 2010, 19(18):3853-3856.

73. Parks $M$, Cronn $R$, Liston $A$ : Increasing phylogenetic resolution at low taxonomic levels using massively parallel sequencing of chloroplast genomes. BMC Biology 2009, 7:84.

74. Neubig K, Whitten W, Carlsward B, Blanco M, Endara L, Williams N, Moore M: Phylogenetic utility of $y c f 1$ in orchids: a plastid gene more variable than matK. Plant Syst Evol 2009, 277(1):75-84.

75. Kuroda H, Maliga P: The plastid clpP1 protease gene is essential for plant development. Nature 2003, 425(6953):86-89.

76. Drescher A, Ruf S, Calsa T, Carrer H, Bock R: The two largest chloroplast genome-encoded open reading frames of higher plants are essential genes. Plant J 2000, 22(2):97-104.

77. Kode V, Mudd EA, lamtham S, Day A: The tobacco plastid accD gene is essential and is required for leaf development. Plant J 2005, 44(2):237-244.

78. Konishi T, Shinohara K, Yamada K, Sasaki Y: Acetyl-CoA carboxylase in higher plants: Most plants other than Gramineae have both the prokaryotic and the eukaryotic forms of this enzyme. Plant Cell Physiol 1996, 37(2):117-122.

79. Gornicki P, Faris J, King I, Podkowinski J, Gill B, Haselkorn R: Plastidlocalized acetyl-CoA carboxylase of bread wheat is encoded by a single gene on each of the three ancestral chromosome sets. Proc Natl Acad SC USA 1997, 94(25):14179-14184.

80. Kubo T, Mikami T: Organization and variation of angiosperm mitochondrial genome. Physiol Plant 2007, 129(1):6-13

81. Knoop $\mathrm{V}$ : The mitochondrial DNA of land plants: peculiarities in phylogenetic perspective. Curr Genet 2004, 46(3):123-139.

82. Sanchez-Puerta MV, Cho Y, Mower JP, Alverson AJ, Palmer JD: Frequent, phylogenetically local horizontal transfer of the cox 1 group I intron in flowering plant mitochondria. Mol Biol Evol 2008, 25(8):1762-1777.

83. Cusimano N, Zhang L-B, Renner SS: Reevaluation of the cox1 group I intron in Araceae and angiosperms indicates a history dominated by loss rather than horizontal transfer. Mol Biol Evol 2008, 25(2):265-276.

84. Bennetzen $\mathrm{JL}$ : The contributions of retroelements to plant genome organization, function and evolution. Trends Microbiol 1996, 4(9):347-353.

85. Kumar A, Bennetzen JL: Plant retrotransposons. Annu Rev Genet 1999, 33:479-532.

86. Vitte $C$, Bennetzen $J$ : Analysis of retrotransposon structural diversity uncovers properties and propensities in angiosperm genome evolution. Proc Natl Acad Sci 2006, 103(47):17638-17643.
87. Vitte C, Panaud O: LTR retrotransposons and flowering plant genome size: emergence of the increase/decrease model. Cytogenet Genome Res 2005, 110(1-4):91-107.

88. Cavagnaro P, Chung S-M, Szklarczyk M, Grzebelus D, Senalik D, Atkins A, Simon P: Characterization of a deep-coverage carrot (Daucus carota L.) BAC library and initial analysis of BAC-end sequences. Mol Genet Genomics 2009, 281(3):273-288.

89. Cavallini A, Natali L, Zuccolo A, Giordani T, Jurman I, Ferrillo V, Vitacolonna N, Sarri V, Cattonaro F, Ceccarelli M, et al: Analysis of transposons and repeat composition of the sunflower (Helianthus annuus L.) genome. Theor App/ Genet 2010, 120(3):491-508.

90. Datema E, Mueller L, Buels R, Giovannoni J, Visser R, Stiekema W, van Ham R: Comparative BAC end sequence analysis of tomato and potato reveals overrepresentation of specific gene families in potato. BMC Plant Biol 2008, 8(1):34..

91. Whiteford N, Haslam N, Weber G, Prugel-Bennett A, Essex J, Roach P, Bradley M, Neylon C: An analysis of the feasibility of short read sequencing. Nucl Acids Res 2005, 33(19):e171.

92. Rogers SO, Bendich AJ: Ribosomal-RNA genes in plants - Variability in copy number and in the intergenic spacer. Plant Mol Biol 1987, 9(5):509-520

93. Sastri DC, Hilu K, Appels R, Lagudah ES, Playford J, Baum BR: An overview of evolution in plant 5S DNA. Plant Syst Evol 1992, 183(3):169-181.

94. Hillis DM, Dixon MT: Ribosomal DNA: Molecular evolution and phylogenetic inference. Q Rev Biol 1991, 66(4):411-453.

95. Volkov R, Kostishin S, Ehrendorfer E, Schweizer D: Molecular organization and evolution of the external transcribed rDNA spacer region in two diploid relatives of Nicotiana tabacum (Solanaceae). Plant Syst Evol 1996, 201(1-4):117-129.

96. Borisjuk NV, Davidjuk YM, Kostishin SS, Miroshnichenco GP, Velasco R, Hemleben V, Volkov RA: Structural analysis of rDNA in the genus Nicotiana. Plant Mol Biol 1997, 35(5):655-660.

97. Bena G, Jubier M-F, Olivieri I, Lejeune B: Ribosomal external and internal transcribed spacers: Combined use in the phylogenetic analysis of Medicago (Leguminosae). J Mol Evol 1998, 46(3):299-306.

98. Volkov RA, Komarova NY, Panchuk II, Hemleben V: Molecular evolution of rDNA external transcribed spacer and phylogeny of sect. Petota (genus Solanum). Mol Phylogenet Evol 2003, 29(2):187-202.

99. Maggini F, Gelati MT, Spolverini M, Frediani M: The intergenic spacer region of the rDNA in Olea europaea L. Tree Genet Genom 2008, 4(2):293-298

100. Cronn RC, Zhao XP, Paterson AH, Wendel JF: Polymorphism and concerted evolution in a tandemly repeated gene family: $5 \mathrm{~S}$ ribosomal DNA in diploid and allopolyploid cottons. J Mol Evol 1996, 42(6):685-705.

101. Fujiwara M, Inafuku J, Takeda A, Watanabe A, Fujiwara A, Kohno S, Kubota S: Molecular organization of 5S rDNA in bitterlings (Cyprinidae). Genetica 2009, 135(3):355-365.

102. Saini A, Jawali N: Molecular evolution of 5 S rDNA region in Vigna subgenus Ceratotropis and its phylogenetic implications. Plant Syst Evol 2009, 280(3-4):187-206

103. Stage DE, Eickbush TH: Sequence variation within the rRNA gene loci of 12 Drosophila species. Genome Res 2007, 17(12):000.

104. Ganley ARD, Kobayashi T: Highly efficient concerted evolution in the ribosomal DNA repeats: Total rDNA repeat variation revealed by wholegenome shotgun sequence data. Genome Res 2007, 17(2):184-191.

105. Kolosha VO, Fodor I: Nucleotide sequence of Citrus limon $26 \mathrm{~S}$ ribosomalRNA gene and secondary structure model of its RNA. Plant Mol Biol 1990, 14(2):147-161.

106. Nei M, Rooney AP: Concerted and birth-and-death evolution of multigene families*. Annu Rev Genet 2005, 39(1):121-152.

107. Baxter I, Tchieu J, Sussman MR, Boutry M, Palmgren MG, Gribskov M, Harper JF, Axelsen KB: Genomic comparison of P-Type ATPase ion pumps in Arabidopsis and Rice. Plant Physiol 2003, 132(2):618-628.

108. Thomson RC, Wang IJ, Johnson JR: Genome-enabled development of DNA markers for ecology, evolution and conservation. Mol Ecol 2010, 19(11):2184-2195.

doi:10.1186/1471-2164-12-211

Cite this article as: Straub et al.: Building a model: developing genomic resources for common milkweed (Asclepias syriaca) with low coverage genome sequencing. BMC Genomics 2011 12:211. 